\title{
Investigating added value of regional climate modeling in North American winter storm track simulations
}

\author{
E. D. Poan ${ }^{1} \cdot$ P. Gachon ${ }^{2} \cdot$ R. Laprise ${ }^{1} \cdot$ R. Aider ${ }^{3} \cdot$ G. Dueymes $^{1}$
}

Received: 5 August 2016 / Accepted: 2 May 2017 / Published online: 31 May 2017

(c) The Author(s) 2017. This article is an open access publication

\begin{abstract}
Extratropical Cyclone (EC) characteristics depend on a combination of large-scale factors and regional processes. However, the latter are considered to be poorly represented in global climate models (GCMs), partly because their resolution is too coarse. This paper describes a framework using possibilities given by regional climate models (RCMs) to gain insight into storm activity during winter over North America (NA). Recent past climate period (1981-2005) is considered to assess EC activity over NA using the NCEP regional reanalysis (NARR) as a reference, along with the European reanalysis ERA-Interim (ERAI) and two CMIP5 GCMs used to drive the Canadian Regional Climate Model-version 5 (CRCM5) and the corresponding regional-scale simulations. While ERAI and GCM simulations show basic agreement with NARR in terms of climatological storm track patterns, detailed bias analyses show that, on the one hand, ERAI presents statistically significant positive biases in terms of EC genesis and therefore occurrence while capturing their intensity fairly well. On the other hand, GCMs present large negative intensity biases in the overall NA domain and particularly over NA eastern coast. In addition, storm occurrence over the northwestern topographic regions is highly
\end{abstract}

E. D. Poan

emmanuel.poan@gmail.com

1 Dép. des Sciences de la Terre et de l'atmosphère, Centre Pour l'Étude et la Simulation du Climat à l'Échelle Régionale (ESCER), Université du Québec à Montréal (UQAM), PO Box 8888, Stn. Downtown, Montréal, Québec H3C 3P8, Canada

2 Dép. de Géographie, ESCER Research Centre, University of Québec at Montréal, Québec, Canada

3 Recherche en Prévision Numérique, Environment and Climate Change Canada, Montréal, Québec, Canada overestimated. When the CRCM5 is driven by ERAI, no significant skill deterioration arises and, more importantly, all storm characteristics near areas with marked relief and over regions with large water masses are significantly improved with respect to ERAI. Conversely, in GCMdriven simulations, the added value contributed by CRCM5 is less prominent and systematic, except over western NA areas with high topography and over the Western Atlantic coastlines where the most frequent and intense ECs are located. Despite this significant added-value on seasonalmean characteristics, a caveat is raised on the RCM ability to handle storm temporal 'seriality', as a measure of their temporal variability at a given location. In fact, the driving models induce some significant footprints on the RCM skill to reproduce the intra-seasonal pattern of storm activity.

Keywords Regional climate model $\cdot$ Added value $\cdot$ Storm tracking $\cdot$ Temporal seriality

\section{Introduction}

Extratropical Cyclones (ECs) account for a large fraction of mid-latitude rainfall as well as extreme and severe weather events such as heavy precipitation, snow storms, coastal waves and flooding, which are responsible for important socio-economic and human damage (e.g. Liberato 2014). Pfahl and Wernli (2012) have shown that, over the eastern half of Northern America, more than $60 \%$ of the daily extreme precipitation (i.e. daily events with intensity above the 99th percentile) are coincident with ECs, and this number increases northeastward, reaching $80 \%$ over the Canadian Maritimes.

EC dynamics is primarily explained by the presence of large-scale baroclinic energy due to strong horizontal 
temperature gradients between polar and tropical air masses in mid-latitudes (e.g. Sanders and Gyakum 1980; Chang et al. 2002). Other regional/small scale processes that merit consideration are lee cyclogenesis (cyclone development on the leeward side of topography) and coastal cyclogenesis (in the presence of a large land-sea temperature contrast, such as between the cold North American continent and the warm waters of the Gulf Stream). Among others, Stull (2000) has stressed the importance of moisture availability, while Gachon et al. (2003) have highlighted the importance of the local temperature Laplacian and sensible heat fluxes (e.g. along the sea-ice margin in winter months) in increasing vorticity and therefore storm growth rate.

Representing these features in models is challenging from both weather and climate perspectives. For instance, Colle et al. (2015) have reviewed historical and future changes of EC activity along the East Coast of the USA and have pointed out that global climate models (GCMs) have difficulty in capturing the role of certain key features such as sea surface temperature (SST) gradients, latent heat release within storms, and also dynamical interactions with the jet stream. However, recent studies using GCMs from the 5th phase of the Coupled Model Inter-comparison Project (CMIP5) have shown quite reasonable climatology in storm track patterns with respect to reanalysis products (e.g. Seiler and Zwiers 2016; Ulbrich et al. 2008). In general, the comparison between GCMs and reanalyses has often shown negative bias for the intensity of ECs (e.g., Zappa et al. 2013), considerable dispersion when assessing intense to extreme storm events (Lambert and Fyfe 2006; Seiler and Zwiers 2016), and weaker cyclogenesis (Bengtsson et al. 2006; Pinto et al. 2006).

The coarse horizontal resolution of most GCMs providing century-long climate simulations may present an important limitation for simulating realistic EC occurrences and intensities. For instance, Jung et al. (2006) have shown that, at spectral truncation of T95, only $60 \%$ of observed storms are detected, while Colle et al. (2013) found that 6 of the 7 CMIP5 GCMs with resolution finer than $1.5^{\circ}$ were able to better capture storm track maxima just north of the Gulf Stream, and to the east of southern Greenland. The resolution effect has also been invoked for weather reanalysis storm tracking. For instance, Trigo (2006) and Allen et al. (2010) have shown marked discrepancies in the number of storms detected when using the NCEP/NCAR (National Centers for Environmental Prediction/National Center for Atmospheric Research) reanalysis at $2.5^{\circ}$ versus the ECMWF (European Centre for Medium range Weather Forecasts) 40 -year one at $1.125^{\circ}$ of horizontal resolution. On the other hand, Hodges et al. (2011) have shown smaller but still significant differences over North America (NA) between relatively fine resolution reanalyses, such as the ECMWF ERA-Interim reanalysis at $0.75^{\circ}$ and the NCEP CFSR (Climate Forecast System Reanalysis) at $0.5^{\circ}$.

To improve our understanding of storm activity and interactions with regional/local features, the present study takes advantage of finer grid-mesh datasets $\left(0.44^{\circ} \times 0.44^{\circ}\right.$ model of the Coordinated Regional Climate Downscaling Experiment CORDEX - Giorgi et al. 2009) over North American simulations. This allows the added value (AV) of dynamical downscaling to be assessed with regard to EC characteristics such as occurrence, intensity, cyclo-genesis and -lysis regions over NA and oceanic boundaries (e.g., Pacific and Atlantic coasts). The Canadian Regional Climate Model (RCM) - version 5 (CRCM5) is used for this purpose. In addition, an objective storm-tracking algorithm described in Sinclair $(1994,1997)$ is used to identify storm tracks and retrieve their parameters. This algorithm is part of the recent international initiative to compare various tracking methods under the Intercomparison of mid latitude storm diagnostics (IMILAST; Neu et al. 2013) project.

Direct comparisons between global and dynamically downscaled data can lead to systematic biases. Côté et al. (2015) have shown that evaluating EC activity in RCM against global data presents multiple challenges, including the sensitivity of cyclogenesis and cyclolysis to boundary effects. Nevertheless, a few recent studies have used RCM to analyze storm activity over the Western Atlantic Coast of North America (WAC) and the North Atlantic (e.g. He et al. 2013; Marciano et al. 2015). Long et al. (2009) have shown that an earlier version of the Canadian Regional Climate Model could improve the representation of intense cyclones with respect to its driving GCM. Among other interesting points, RCMs provide an improved description of the surface topography which, in the case of North America, is a decisive element in storm development (Brayshaw et al. 2009). While RCMs are reasonably appealing, it should not be forgotten that these models are driven by coarser datasets and therefore might inherit some biases from their boundary conditions. In addition, RCMs carry their own structural biases (Šeparović et al. 2013) due to many approximations, including the parameterization and numerical schemes used. Despite these uncertainties, RCMs may provide GCMs with a useful and complementary tool for climate studies (e.g., Laprise et al. 2003; Laprise 2008) and, in particular, can help improve our comprehension and representation of storm activity. This is partly confirmed by the recent study by Colle et al. (2015), which pointed out that only $5-10 \%$ of cyclone densities are unpredicted over the West Atlantic when the ensemble RCM runs from the NARCCAP (North American Regional Climate Change Assessment Program-Mearns et al. 2009) project are used.

Finally, EC temporal 'seriality', described in Mailier et al. (2006) as the rate at which storms transit through a 
given location, is a crucial aspect that needs to be well represented in climate models. Over the eastern Atlantic, temporally clustered activity (larger than average storm transits at a specific location) is often considered as a potential source of socio economic damage. For example, between 26th and 28th December 1999, two successive intense storms named Lothar and Martin (Ulbrich et al. 2001; Wernli et al. 2002; Goyette et al. 2003) transited over Europe, leading to about 130 human deaths and 13 billion Euros of economic losses. Conversely, over the western Atlantic, because of the quasi-permanent and strong winter baroclinic energy, regular (i.e. persistent) storminess can be as damaging as clustered storms. Pinto et al. (2013) recently addressed the question of 'seriality' with regard to the current and future climate in mid-latitudes. They found that storm occurrences tended to be a regular process rather than being clustered at the entrance of the North Atlantic storm track. On the other hand, the same study showed that GCM projections suggested a change toward more clustered patterns over the WAC, consistent with the overall northward migration of the baroclinic zone. Vitolo et al. (2009) have shown that storms tend to be more clustered when only intense storms are considered (i.e. the 90th percentile). This paper gives an opportunity to gain more insight into the issue of EC temporal seriality over the NA domain and from reanalysis, RCM and GCM perspectives. In particular, regional storminess regimes (regular-intermittent-highly active) will be analyzed.

The paper is organized as follows. Data and methodology are described and discussed in Sect. 2. Section 3 assesses the climatology of storm characteristics in reanalyses and GCMs, and in CRCM5 simulations driven with different boundary conditions. Section 4 elaborates on the regional structure of storm temporal 'seriality' over known NA areas of active cyclogenesis. The main findings are summarized in Sect. 5, together with suggestions for further developments.

\section{Data and methodology}

\subsection{Data: description of simulations}

\subsubsection{Reanalysis data}

In the present study, two sets of reanalysis products are used:

1. NCEP North American Regional Reanalysis (NARR; Mesinger et al. 2006), available from 1979 to present, generated by a regional model nested in the NCEPDOE (Department of Energy) global model. NARR is generated using the very high resolution NCEP Eta
Model (32-km with 45 vertical levels) together with the Regional Data Assimilation System that, significantly, assimilates precipitation along with the other atmospheric variables. Due to improvements in the model/assimilation system, NARR temperature, winds and precipitation are substantially better than those given by NCEP-DOE. Because of its finer resolution $\left(0.25^{\circ} \times 0.25^{\circ} ; 3\right.$ hourly $)$, which is more relevant for regional (scale) studies, NARR will be considered as the reference dataset in our analysis.

2. The ECMWF reanalysis ERA-Interim (hereafter ERAI; Dee et al. 2011), available from 1979 to the present, is a global reanalysis computed with the spectral horizontal resolution T255 $(\sim 80 \mathrm{~km})$ and with 60 levels in the vertical. Data are archived on a 6 hourly basis. ERAI has been used by many studies to assess models (CMIP5) as well as in comparisons with other reanalysis datasets (e.g., Colle et al. 2013). Coté et al. (2015) have noted, however, that its relatively smooth topography over the Rockies can lead to overestimated cyclonic activity over western North America.

\subsubsection{GCM and RCM simulations}

Two GCMs taking part in the CMIP5 project and used for IPCC assessment reports (e.g., IPCC 2013) were used to provide boundary conditions for the RCM runs:

1. The Canadian Earth System Model, version 2CanESM2 (Arora et al. 2011) is a global climate model developed by the Canadian Centre for Climate Modelling and Analysis (CCCma) of Environment and Climate Change Canada. The CanESM2 atmospheric component-CanAM4 (Canadian Atmospheric Model 4, von Salzen et al. 2013) is a spectral model employing T63 triangular truncation with physical tendencies calculated on a $\sim 2.81^{\circ}$ linear transform grid. CanESM2 historical natural and anthropogenic forcing simulations (1951-2005) are considered here to use direct atmospheric fields for the storm tracking analysis and also to drive the RCM model for both the atmospheric and oceanic boundary conditions.

2. The Max Planck Institute Earth System Model-MPIESM-LR (Giorgetta et al. 2013) is a global atmosphere, ocean and land surface coupled model. For the atmosphere section, the low-resolution version is available at $\mathrm{T} 63 / 1.9^{\circ}$ and will be used over the historical period 1951-2005.

Finally, the Canadian Regional Climate Model version 5-CRCM5 is used to simulate atmospheric regional conditions following the protocol defined by the CORDEX 
project. CRCM5 combines parts of the physics package of the Environment Canada Global Environmental Multiscale (GEM) forecast model (Côté et al. 1998a, b) version 3 (GEM3), but uses the Canadian land-surface scheme CLASS 3.5 (Verseghy 2008) and the interactive coupled model "Flake" to account for lakes (Martynov et al. 2010). ERAI, CanESM2 and MPI-ESM-LR are used to provide three different (atmospheric and oceanic) boundary conditions to run the CRCM5 model using a $0.44^{\circ}$ horizontal grid mesh on a rotated pole grid (see descriptions in Martynov et al. 2013; Šeparović et al. 2013). CRCM5 data are provided on a 3 hourly basis. Finally, it is worth mentioning that no spectral nudging has been applied when conducting the CRCM5 dynamical downscaling experiments.

Table 1 gives details of all the datasets used in this study. Before the storm-tracking algorithm (described in the next section) was run, simulations were all interpolated on a common 100-km polar stereographic (PS100) projection with an identical spatial extent. This was done for both global- and regional-scale simulations extracted over the regional domain of interest (see Fig. 1). As shown by Eichler and Gottschalck (2013), storm tracking is sensitive to the dataset spatial resolution and re-gridding the simulations to a "GCM-RCM intermediate resolution" (i.e. $100 \mathrm{~km}$ ) is believed to help reduce this sensitivity. Because our focus is on synoptic-scale ECs, this intermediate resolution should be able to capture all major winter events regardless of the original resolution. Finally, even though differences in time resolution can play a significant role in EC characteristics (see Blender and Schubert 2000), simulations will be kept at their initial resolutions (i.e. 3 hourly for regional products as NARR and CRCM5, and 6 hourly

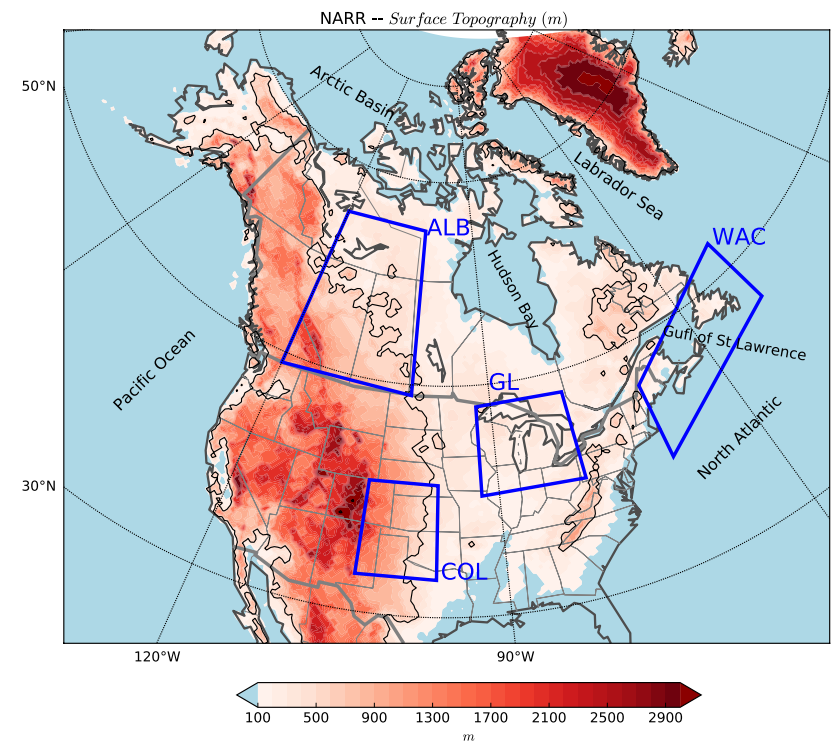

Fig. 1 NARR North America surface topography at $0.25^{\circ}$ over the domain used to track the ECs. The solid black line contours elevation above $500 \mathrm{~m}$. Regions of interest for the study are delimited by the 4 boxes: $A L B$ Alberta, $C O L$ Colorado, GL Great Lakes, WAC Western Atlantic Coast

for the rest, i.e. available archived fields common to all global reanalysis and GCMs).

\subsubsection{Oceanic boundary conditions}

To diagnose the ocean related baroclinic energy (e.g., Branscome et al. 1989) provided by the boundary conditions (reanalysis and GCMs), SSTs are evaluated. Seiler

Table 1 Data sets used for the storm tracking

\begin{tabular}{|c|c|c|c|c|c|}
\hline Simulation function & Simulation type & Simulation short name & $\begin{array}{l}\text { Native time/ } \\
\text { space resolu- } \\
\text { tion }\end{array}$ & Time period/domain & Long name/Institution \\
\hline Reference & Regional Reanalysis & NARR & $0.25^{\circ} / 3$ hourly & $\begin{array}{l}\text { 1979-2012/ North } \\
\text { America (NA) }\end{array}$ & $\begin{array}{l}\text { NCEP North American } \\
\text { Regional Reanalysis (USA) }\end{array}$ \\
\hline \multirow[t]{3}{*}{ Driving data } & Global Reanalysis & ERAI & $0.75^{\circ} / 6$ hourly & 1961-2012/Global & $\begin{array}{l}\text { European Centre for Medium } \\
\text { range Weather Forecast } \\
\text { Reanalysis (EU) }\end{array}$ \\
\hline & \multirow[t]{2}{*}{ GCMs } & CanESM2 & $1.875^{\circ} / 6$ hourly & 1961-2005/ NA & $\begin{array}{l}\text { Canadian Earth System } \\
\text { Model-Environment and } \\
\text { Climate Chance Canada } \\
\text { (ECCC-CANADA) }\end{array}$ \\
\hline & & MPI-ESM-LR & $1.875^{\circ} / 6$ hourly & 1961-2005/ NA & $\begin{array}{l}\text { Max Planck Institute Earth } \\
\text { System Model (GERMANY) }\end{array}$ \\
\hline Regional climate model & $\mathrm{RCM}$ & CRCM5 & $0.44^{\circ} / 3$ hourly & 1961-2012/NA & $\begin{array}{l}\text { Canadian Regional Climate } \\
\text { Model - Université du } \\
\text { Québec à Montréal (UQAM- } \\
\text { CANADA) }\end{array}$ \\
\hline
\end{tabular}

See text for details about climatological time period and native resolutions 
and Zwiers (2016) have shown that GCM skill is strongly impacted by SSTs and the related upper tropospheric jets. For NARR, over open water (seas and lakes), SSTs are derived from $1^{\circ}$ SST observations by Reynolds et al. (2002) while, when sea/lake ice is detected (e.g. frozen lakes or sea), SSTs stand for the surface skin temperature derived from the energy balance equation (see Mesinger et al. 2006). For ERAI, the prescribed boundary conditions are taken from different sources (Kumar et al. 2013) as follows: from January 1989 to June 2001, NCEP 2D-Var SSTs; from July 2001 to December 2001 NOAA (National Oceanic and Atmospheric Administration) Optimum Interpolation SSTs; from January 2002 to January 2009, NCEP Real-Time Global SSTs; and from February 2009 onwards, the Met Office Operational SSTs and Sea-Ice Analysis (OSTIA). As in NARR, the open ocean SSTs are derived from these observations. However, in the presence of sea ice, ERAI SSTs are assumed to be $-1.7^{\circ} \mathrm{C}$, corresponding to the average temperature of frozen seawater (at a salinity of $34 \%$ ). The 2 GCMs use SSTs from their oceanic modeling component where frozen SST is set constant and close to $-1.7^{\circ} \mathrm{C}$. Since the analysis is done during the winter, the northern Canadian seas/lakes are mostly frozen and large differences are expected from NARR and the other three datasets. Therefore, comments related to SST structure are limited to open water areas.

\subsection{Methodology}

\subsubsection{Storm tracking algorithm: detection criteria and tracking approach}

A modified version of Sinclair's $(1994,1997)$ storm-tracking algorithm was used to retrieve individual cyclone trajectories for all simulations described in Table 1. Actually, Sinclair (1997) used the 1000-hPa geopotential heights to compute the vorticity field. However, the NA topographic features (higher than $1000 \mathrm{~m}$ over the western half of the region in Fig. 1) make it hard and rather inconvenient to use this level, particularly over the western side. Also, GCM fields are not systematically archived at $1000 \mathrm{hPa}$, especially the geopotential height or wind fields. For a more convenient and consistent evaluation of ECs over NA, atmospheric variables at 850 -hPa will be used. The tracking algorithm has also benefitted from several years of development at UQAM (University of Quebec At Montréal; e.g., Rosu 2005; Radojevic 2006), but only the main steps (originally from Sinclair's previous work) are reviewed here:

1. $850 \mathrm{hPa}$ winds are spatially derived to obtain the relative vorticity $\left(\boldsymbol{\zeta}_{r}\right)$ and this latter variable is spatially smoothed to remove smaller scale features or additional biases due to original grid resolution that is significantly different between GCMs and RCMs. To do so, a Cressman's filter is used to average data at each grid point with neighboring grid points within a $800 \mathrm{~km}$ radius circle.

2. $\zeta_{r}$ local maxima exceeding the $1.5 \mathrm{CVU}$ (cyclonic vorticity unit, $10^{-5} \mathrm{~s}^{-1}$ ) are taken as cyclonic centers (CC) for the tracking process. A bicubic spline fit is used in order to locate the CC more accurately.

3. Many detected CC are stationary orographic features resulting from lee troughs or heat lows. These spurious centers are eliminated by requiring centers to have a total displacement of at least $1200 \mathrm{~km}$ in $24 \mathrm{~h}$, equivalent to a horizontal speed of about $50 \mathrm{~km} \mathrm{~h}^{-1}$ (often observed for winter synoptic scale disturbances over NA; Arhens 2009)

4. EC life cycle construction is based on probabilistic functions involving the previous/current/next positions of the CC and the 500-hPa wind modulus. It is commonly accepted as a 'forecaster rule' in meteorology that storms move approximately along the $500-\mathrm{hPa}$ wind and the speed at which surface systems travel is estimated as half the 500-hPa wind speed (e.g., Ahrens 2009). A match is attempted between each of these predictions (location) and the set of nearest centers found a time step later. An ensemble of successfully chosen matches satisfies the condition of minimizing a cost function, which is a weighted sum of absolute departures of position, pressure and vorticity from predicted values (Murray and Simmonds 1991; Sinclair 1994).

5. The variables retrieved from the tracking are representative of a reference surface of $2.5^{\circ} \times 2.5^{\circ}$ (defined as $1 \mathrm{SU}$, surface unit) and are defined as follows: (1) Cyclone occurrence, also often referred to as frequency or track density, which is the number of 3-hourly (or 6-hourly) CC detected and associated with a track (unit = \#CC SU${ }^{-1}$ month $^{-1}$ ); (2) Cyclone intensity, which is the relative vorticity (in CVU) associated with the CC, (3) Cyclone genesis and lysis rates (\#CC $\mathrm{SU}^{-1}$ month $^{-1}$ ), which simply refer to the first and last points in the cyclone track respectively.

It is worth mentioning that, in the present study, "winter season" refers to the northern hemisphere cold season extending from November to March and will be noted NDJFM. Model skill will be primarily compared with NARR but also with ERAI in order to link the current results with previous studies. Statistical significance tests will be performed in the sense of Mann-Whitney-Wilcoxon tests (Wilcoxon 1945) at the 99\% significance level. 


\subsubsection{Winter storm temporal seriality}

To study winter storm temporal 'seriality', an approach similar to that of Mailier et al. (2006) was used, as in the recent work over the North Atlantic by Pinto et al. (2013). If cyclone occurrences were completely random (i.e., the occurrence of one cyclone at any moment is independent of previous cyclone occurrences), its distribution could be described by a one-dimensional Poisson process with a constant rate or intensity (=seasonal mean at each location). Two alternative hypotheses to complete randomness are possible: (1) serial clustering, according to which the passage of one cyclone could trigger other cyclones as the energy propagates downstream, and (2) serial regularity, which may arise if favorable background conditions help to maintain a regular rate of cyclogenesis and the resulting cyclones are permitted to occur within a minimum time-space distance. To verify these hypotheses, the monthly occurrence $(N)$ time series (at each grid point) are considered and a storm dispersion index $\varphi$ is computed as follows (see Pinto et al. 2013):

$\varphi=\frac{\operatorname{Var}(N)}{E(N)}-1$

where $\operatorname{Var}(N)$ stands for the monthly temporal variance and $E(N)$ the monthly mean. A parameter defined in this way compares storm dispersion to a Poisson's process with dispersion 1. Following Eq. (1), the three previously defined hypotheses can be expected:
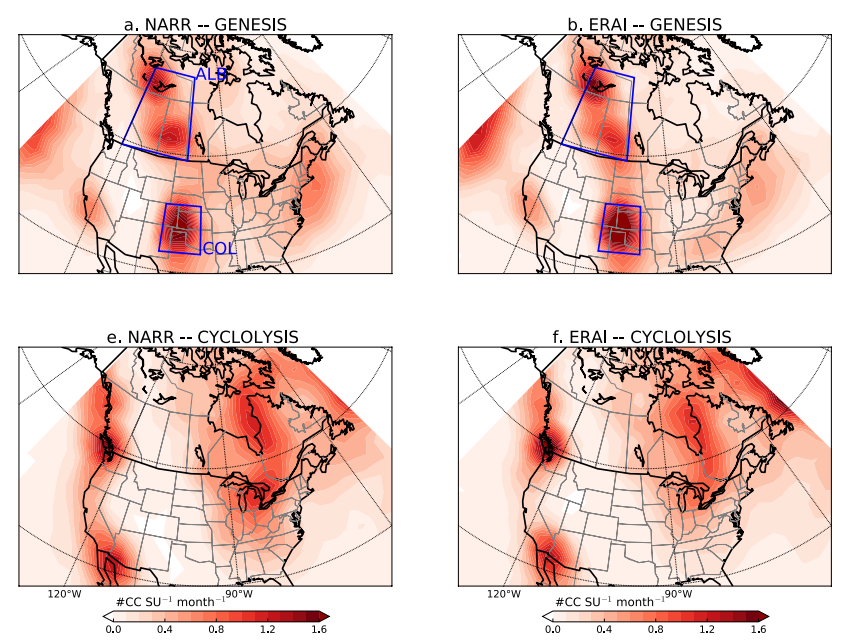

Fig. 2 25-year (1981-2005) NDJFM climatology of storm genesis (top panels) and lysis (bottom panels) rates in [\#CC SU${ }^{-1}$ month $^{-1}$ ] (i.e. cyclonic centers per surface unit SU; $1 \mathrm{SU}=2.5^{\circ} \times 2.5^{\circ}$ ) from: a NARR (used as reference), b ERAI, c CanESM2, and d MPI-ESM-LR. A threshold of 1.5 CVU (cyclonic vorticity units, 1
1. $\varphi=0$, monthly counts are randomly distributed $[\operatorname{Var}(N)=E(N)]$, and therefore storm occurrences follow a homogeneous Poisson process.

2. $\varphi>0$, the process is more dispersive than a simple Poisson process and so cyclone occurrences are temporally clustered (i.e. intermittent process).

3. $\varphi<0$, the process is under-dispersive and therefore cyclone transits at the given location tend to be a recurrent or regular process.

\section{Winter storm characteristics}

\subsection{Reanalysis and global climate model simulations}

\subsubsection{Winter cyclone genesis and lysis pattern}

Figure 2 displays spatial structures of storm genesis (top) and lysis (bottom) rates as defined in the "Methodology" section, for NARR and the three driving datasets. According to Neu et al. (2013), these two parameters are the most subject to discrepancy among methods and models. The discussion focuses on the inner domain as cyclogenesis over the Pacific and cyclolysis over the Atlantic are affected by the boundary locations of the regional simulated domain (close to inflow/outflow over the western/eastern domain limits). Consistently with previous studies (e.g., Grise et al. 2013), both NARR and ERAI (Fig. 2a, b) reanalyses highlight two continental regions of cyclone initiation: the genesis of the so-called Alberta Clippers over western Canada (Stewart et al. 1995) and
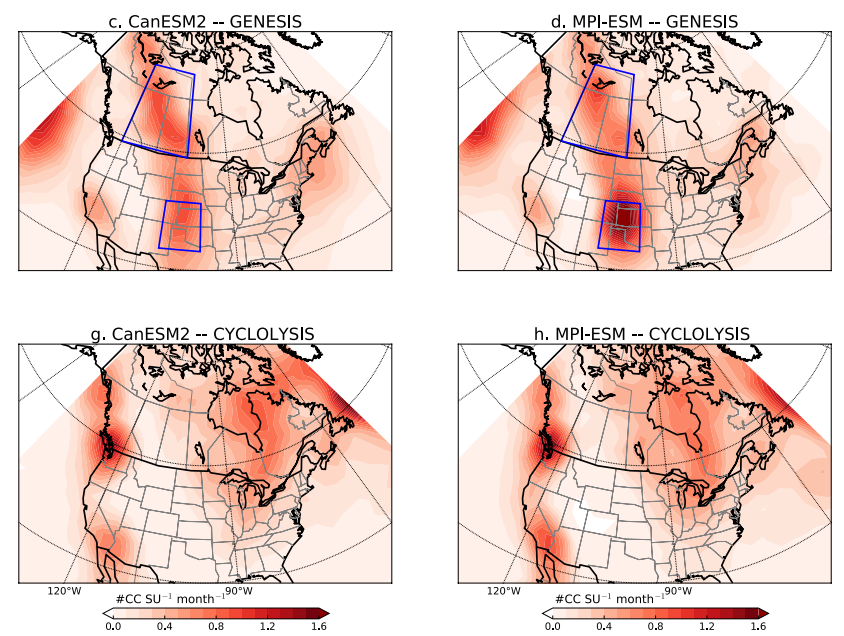

$\mathrm{CVU}=10^{-5} \mathrm{~s}^{-1}$ ) is used for storm detection and tracking while a minimum of $24 \mathrm{~h}$ is required for the lifetime. The Alberta and Colorado regions delimited in a are highlighted for boxplot analysis shown in Fig. 6 
the genesis of the Colorado Lows over the Midwest USA (Sisson and Gyakum 2004). While both reanalyses agree on a stronger genesis rate over Colorado, ERAI tends to overestimate cyclone initiation relative to NARR over this area. In addition to those in Colorado and Alberta, a secondary cyclogenesis region can be seen over the East Coast, with a local maximum near the New England Coast between Cape Hatteras and Cape Cod (well defined in NARR data and underestimated in the ERAI data). As shown by Grise et al. (2013), rather than being a cyclone initiation area, the East Coast region is more subject to a redevelopment or intensification of cyclones because of larger low level baroclinic gradients and also to the presence of diabatic PV (potential vorticity) due to oceanic latent heat release (Lackmann 2011). Therefore, these coastal systems (also called "Nor'easters" by Hirsch et al. 2001) can be quite frequent and explosive ('bombs'), reaching a growth rate that exceeds one bergeron (Seiler and Zwiers 2016) over the western boundary current (i.e. in the vicinity of the main Gulf Stream branch). The GCM realizations show rather poor skill compared to the reanalysis products: CanESM2 (Fig. 2c) strongly underestimates the number of cyclones that form in these three cyclogenesis regions, while MPI-ESM-LR (Fig. 2d)
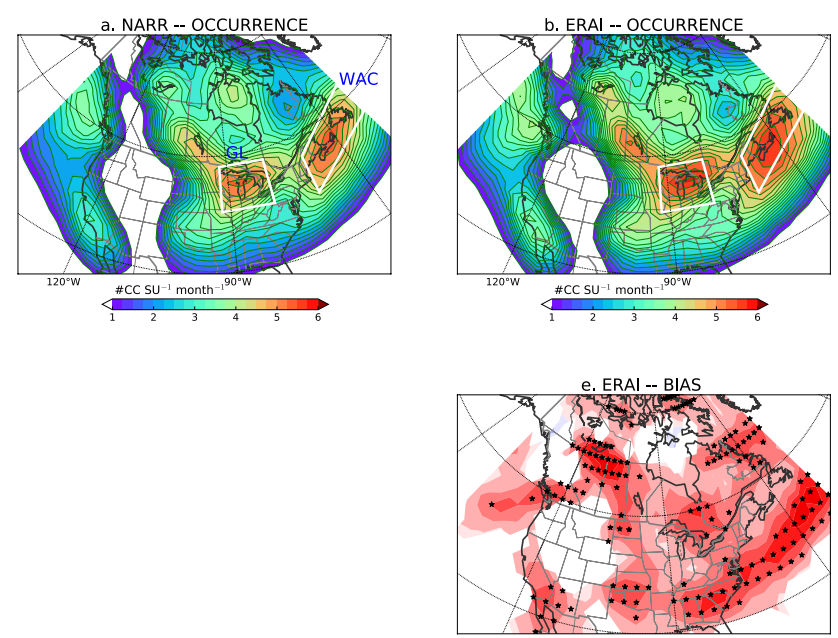

Fig. 3 Same as Fig. 2 but for storm occurrence (top panels). Driving-data occurrence biases with respect to NARR (middle panels) and ERAI (bottom panels) are also shown. Statistically significant differences (biases) in the sense of a Mann-Whitney-Wilcoxon test reproduces more accurately the Colorado Lows genesis rate (as confirmed by the genesis boxplots in Fig. 6i).

The cyclolysis rate (bottom panels in Fig. 2e-h) indicates that the Rocky Mountains act as a strong barrier leading to remarkable cyclolysis over their western limit (over British Columbia and California). There is also a remarkable lysis rate near the eastern sides of continental lakes or inland sea (Great Lakes or Hudson Bay) as the mean environment energy drops drastically after ECs cross from the moist, warm conditions over lakes or open waters to the dry, cold conditions over land. The cyclolysis rate is rather well captured by all four simulations in terms of spatial structures. However, and consistently with their weaker genesis rate, GCMs underestimate cyclolysis at the exit of the Great Lake region and over the eastern part of Hudson Bay, and do not capture the relatively large spatial variability displayed by reanalyses over eastern Canada.

\subsubsection{Winter cyclone occurrence and intensity}

Figures 3 and 4 present the NDJFM mean of storm occurrence and intensity, respectively, over North America for NARR and the three driving datasets. Driving data biases relative to NARR (middle row) and ERAI (bottom) are
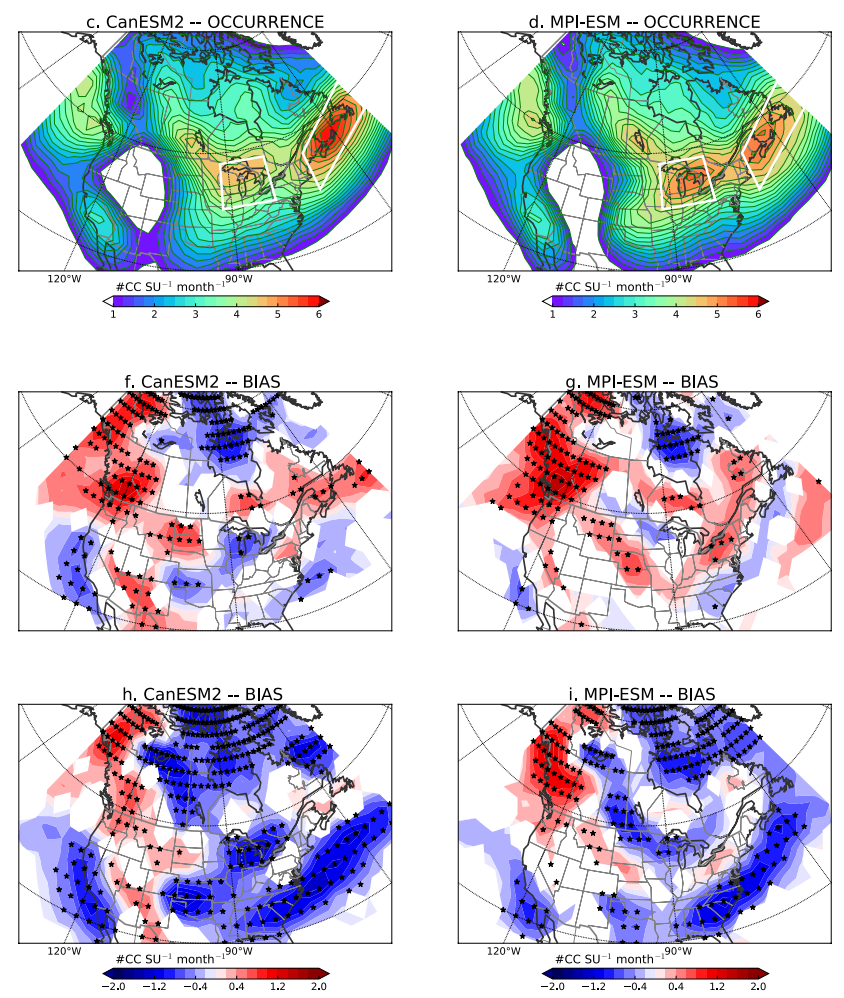

(Wilcoxon 1945) with $p$ value $<0.01$ are shaded by black dots. [\#CC $\mathrm{SU}^{-1}$ month $^{-1}$ ] is the number of cyclonic centers per surface unit $\left(1 \mathrm{SU}=2.5^{\circ} \times 2.5^{\circ}\right)$ per month 

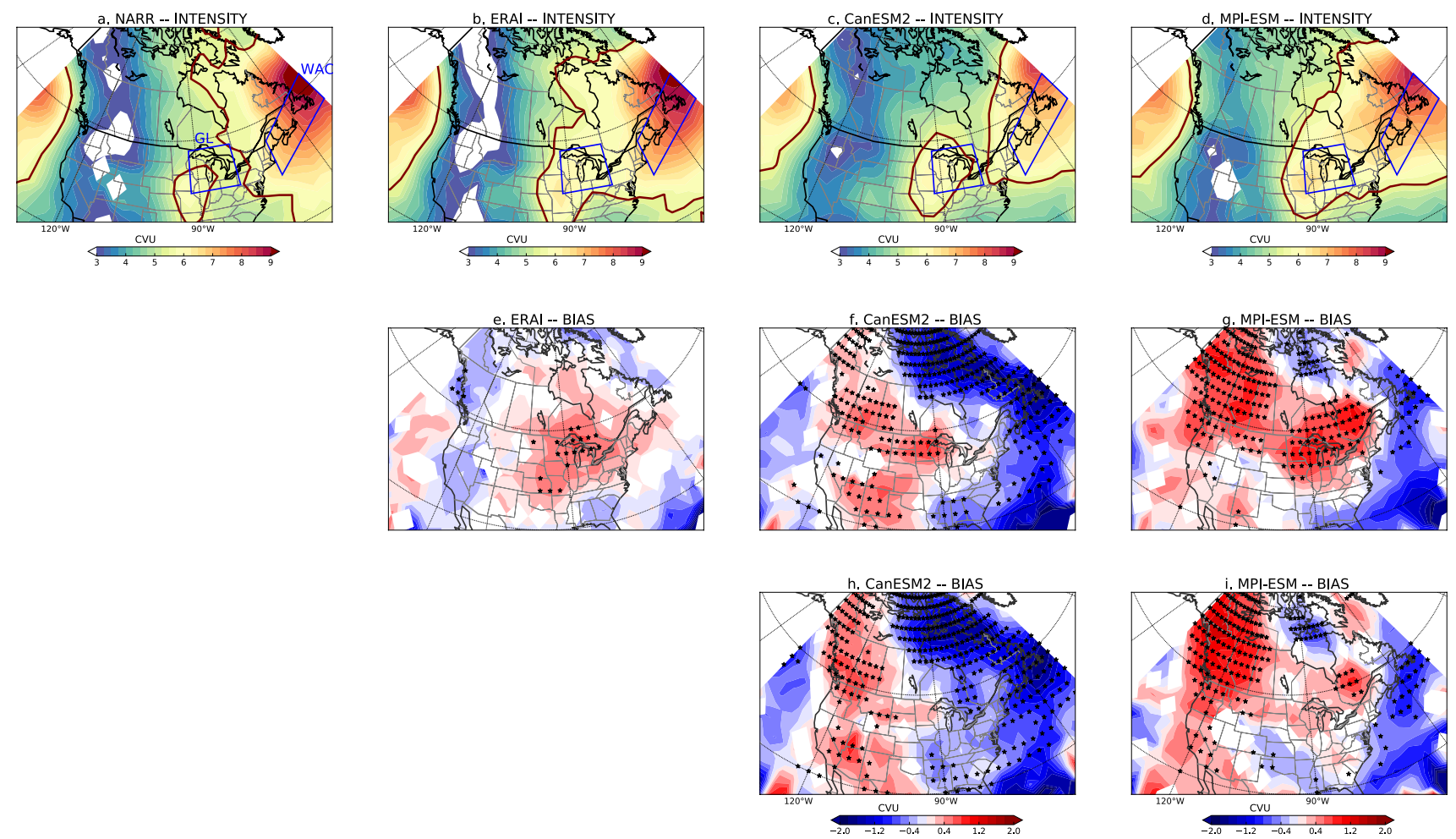

Fig. 4 Same as Fig. 3 but for storm intensity (in CVU). High intensity values (>5 CVU) are delimited with the dark brown contour. The Great Lakes and the Western Atlantic Coast regions are highlighted for occurrence and intensity boxplot analysis shown in Fig. 6

also displayed. All datasets highlight the well-known storm-track pattern over North America marked by a white box over the Great Lakes region and the north Atlantic storm-track entrance over the WAC (Western Atlantic Coast) of the USA and Canadian Maritimes. Historical storm-track classification based on EC origin (Reitan 1974; Hoskins and Hodges 2002 among others) can also be inferred: (1) the West Coast Pacific stormtrack termination over the western Rockies, (2) the leeside tracks from both the Colorado and Canadian Alberta Rockies, merging over the Great Lakes area, and (3) the WAC track following the land-ocean boundaries and reaching an occurrence maximum near Nova Scotia and the eastern side of the Gulf of St. Lawrence. The Hudson Bay region is also marked by high occurrence of storms, well defined by the NARR data (Fig. 3a) and underestimated by the other products (Fig. 3b-d). In fact, Stewart et al. (1995) have shown that, during the winter season, both polar lows and synoptic storms transiting over the St. Lawrence Basin dissipate just after crossing the relatively warm water of Hudson Bay, some even regenerating when open waters are sufficiently large in early winter (e.g., Gachon et al. 2003). On the other hand, a remarkable minimum (or absence) of cyclone occurrence can be seen over the Rocky Mountains, the southern border of the Appalachian Mountains and, to a lesser extent, the Kaniapiskau Plateau in the North of Quebec.

Despite handling the main structures, the three driving datasets, including ERAI, exhibit notable differences with NARR (Fig. 3e-g). The ERAI biases, though surprisingly large, are reminiscent of those of Hodges et al. (2011), where ERAI was shown to overestimate storm occurrence, particularly over northern America, relative to two other reanalyses (NASA-MERRA and NCEP-CFSR) whose resolution is close to that of NARR. It is worth recalling that ERAI occurrences compare well with those detected by Grise et al. (2013) using the same data, but with a different tracking algorithm. Overall, these biases raise the question of which data can be considered as references in model intercomparison exercises.

The 2 CMIP5 GCMs show, consistently with their lower (coarser) topography, a positive bias over the Canadian Rockies, i.e. they overestimate CC over regions with high relief (Fig. 3f, g). In contrast, the Hudson Bay maximum is underestimated. This is likely due to the lack of oceanic grid points, according to their coarse horizontal resolution, inducing an underestimation of regional-scale latent and sensible heat fluxes from open water areas present in early winter. Such fluxes are partly responsible for EC intensification or the development of polar lows (see Gachon et al. 2003). Over the East Coast, occurrence biases are moderate 
for both GCMs. However, when compared with ERAI, consistently with previous studies (e.g., Colle et al. 2013; Seiler and Zwiers 2016; Zappa et al. 2013), GCMs show a large negative occurrence bias near the East Coast and over the Gulf Stream area (Fig. 3c, d, f-i).

Despite differences in their occurrence field, the intensity of winter storms (Fig. 4) is relatively well captured by NARR and ERAI, showing large values ( $>5 \mathrm{CVU}$ ) east of the continental genesis region and reaching a maximum over the Newfoundland area. Bias assessment shows that ERAI slightly overestimates the intensity over the Great Lakes, where surface water temperature can play an important role before the formation of lake-ice. The GCM simulations fail to capture the intensity amplitude. Red areas over the continental regions of NA imply that ECs of both GCMs are too intense with respect to NARR and ERAI. Over the East Coast, the negative bias pattern is very salient (both in terms of location of maximum values and in spatial extent, especially for CanESM2 data). In fact, both GCMs follow the CMIP5 ensemble mean (e.g., Seiler and Zwiers 2016), being barely sensitive to the well-known WAC intense cyclones associated with the warm SSTs (the Gulf Stream water) or along the coast of eastern North America where land/sea temperature contrasts are high.

The SST anomalies shown in Fig. 5 are quite substantial within these GCMs, particularly for CanESM2 and for the North Atlantic. Along the land/sea boundary, SST gradients constitute a good proxy when diagnosing the potentiality of storminess (Nakamura et al. 2004;
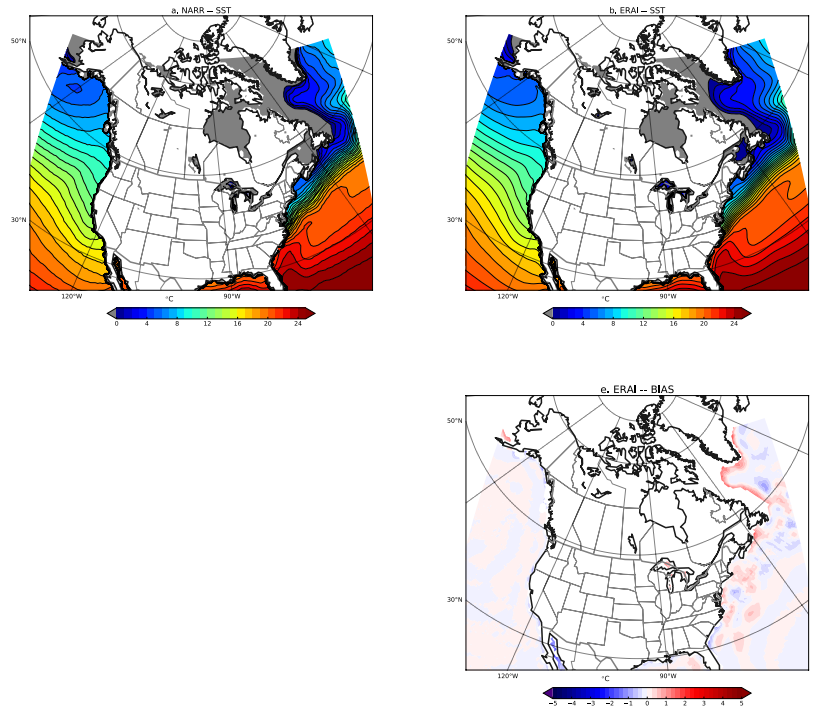

Fig. 5 25-year NDJFM mean SST $\left({ }^{\circ} \mathrm{C}\right)$ from NARR (a) and the 3 driving (ERAI, CanESM2, and MPI-ESM-LR) datasets (b-d, respectively). The bottom panels represent climatological bias with respect to NARR from ERAI (e), CanESM2 (f) and MPI-ESM-LR (g), respectively. Over the open ocean, SSTs represent the sea surface
Hoskins and Valdes 1990). Boundary conditions brought by SST features (amplitudes, spatial and temporal variability) can significantly impact the ability of a model to generate EC initiation, and then its development and/ or intensification for those arriving from continental regions. Woollings et al. (2010) have shown that SST spatial and temporal resolutions (considering that they correspond to prescribed values over open water areas in RCM), strongly influence the ability of an RCM (and a GCM) to capture the key features of storms. Colle et al. (2015) have concluded that the question of whether it is SST values or their gradients that influence storms most is still open. Similarly, Booth et al. (2012) have shown that storm strength increases monotonically with SST magnitudes, even for weak SST gradients, and have concluded that "the SST beneath the storm can have just as important a role as the SST gradients in local forcing of the storm". With such warm biases of SSTs, GCMs would be expected to provide more humidity and thus diabatic heating (latent and sensible) PV, which, in turn, should increase storm development (Bluestein 1993). Instead, SST does not appear to be such a decisive component in determining GCM ability to simulate EC characteristics, i.e. at the scale of a GCM. Zappa et al. (2013) have consistently shown that prescribing "observed" SSTs as boundary conditions in CMIP5 GCMs does not significantly suppress this negative bias in intensity or EC occurrence over the WAC. Ultimately, the lack of fine spatial, and particularly temporal, resolution for SST
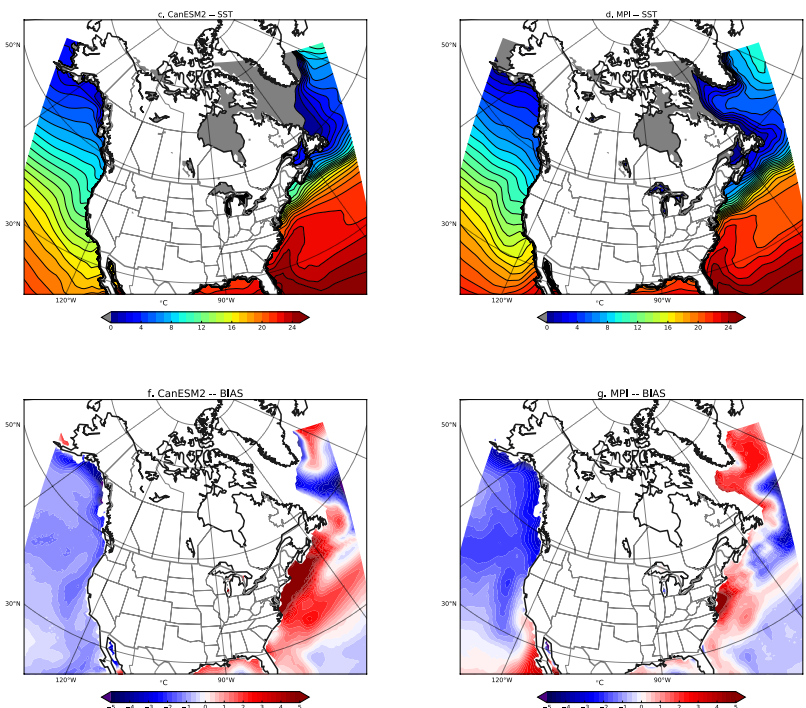

temperatures for all datasets while, over frozen areas, SST definition may vary. See text for more details. Gray shaded areas correspond to the presence of sea-ice in March (maximum extent during the November to March period) for each dataset 
seems to be the key factor preventing improved climate model skill with respect to coastal EC characteristics. This was demonstrated, at least at the short-term synoptic scale, over the Gulf of St. Lawrence area in early winter, where the high frequency (high resolution) coupling between atmosphere and ocean has quite a substantial impact on the accuracy of meteorological forecasts over the whole area, especially when ECs are involved (Gachon and Saucier 2003; Pellerin et al. 2004). In fact, the high resolution and frequency interactions between atmosphere and surface oceanic conditions from the fall to winter months can strongly modify SST and sea-ice conditions at the daily scale (e.g., Gachon et al. 2001), leading to substantial modification in the surface and the air temperature gradients (see Bourassa et al. (2013) for a related discussion on turbulent surface fluxes and air/ ocean and sea-ice interactions). All these surface forcing factors and potential SST biases play a key role in EC development and intensification at high resolution over oceanic areas, i.e. from the RCM simulations analyzed below.
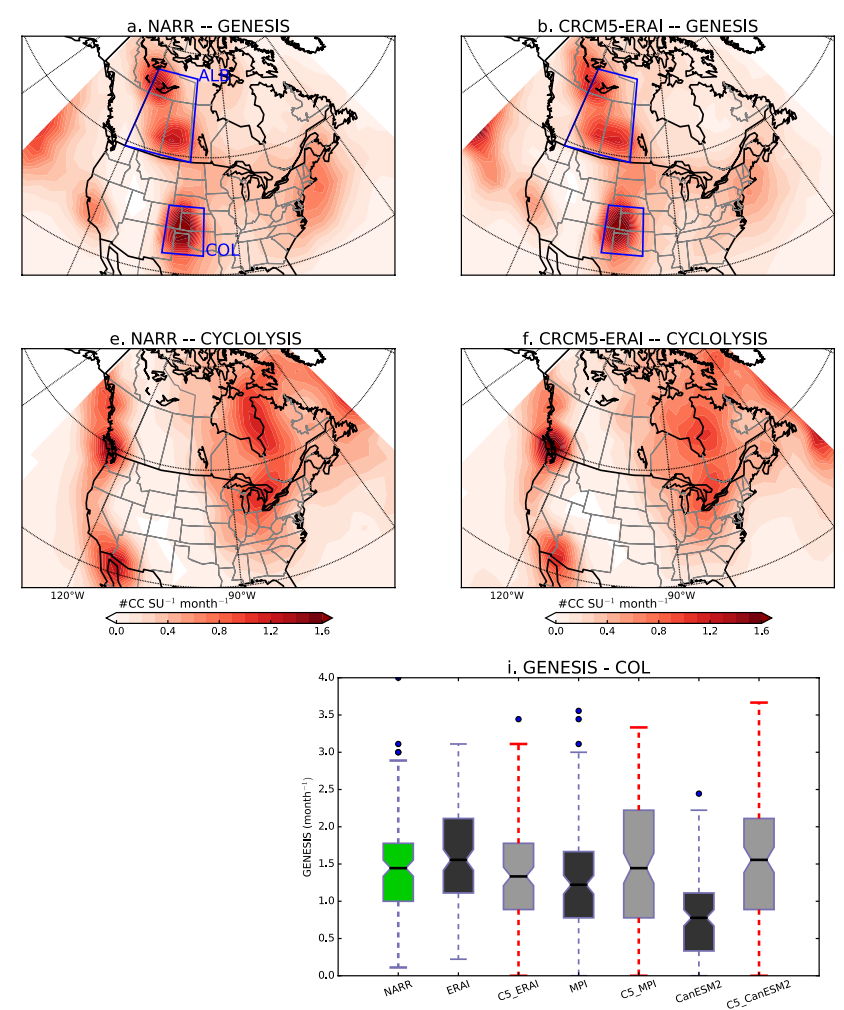

Fig. 6 Top (a-h) same as Fig. 2a, e but for CRCM5 simulations driven by ERAI (b, f), CanESM2 (c, g), and MPI-ESM-LR (d, h) respectively. Bottom $(\mathbf{i}, \mathbf{j})$ 25-year NDJFM monthly storm genesis boxplot for all simulations over the Colorado (i) and Alberta (j) regions (see NARR top panel for their respective locations). The reference NARR is in green, driving data are in black and the cor-

\subsection{Assessment of CRCM5 storm characteristics}

\subsubsection{Storm genesis and lysis}

In the following, the analysis seeks to assess the regional model structural error occurring when the model is driven by the most accurate available boundary conditions (i.e. reanalysis ERAI products) and, secondly, the value added by regional modeling (Di Luca et al. 2015) with respect to global climate simulations (MPI-ESMLR and CanESM2) also used as boundary conditions. Figure 6 displays winter storm genesis (Fig. 6a-d) and lysis (Fig. 6e-h) for NARR (for easier comparison) and the 3 simulations of CRCM5. In addition, genesis rate boxplots computed for the Colorado (Fig. 6i) and the Alberta (Fig. 6j) regions are displayed. It is worth recalling that the boxplots do not include spatial variability since the genesis variable is spatially averaged, so statistics are computed over the monthly time series. ERAI-driven CRCM5 (Fig. 6b) captures the genesis pattern exhibited by NARR remarkably, outperforming ERAI over both continental initiation areas (Colorado
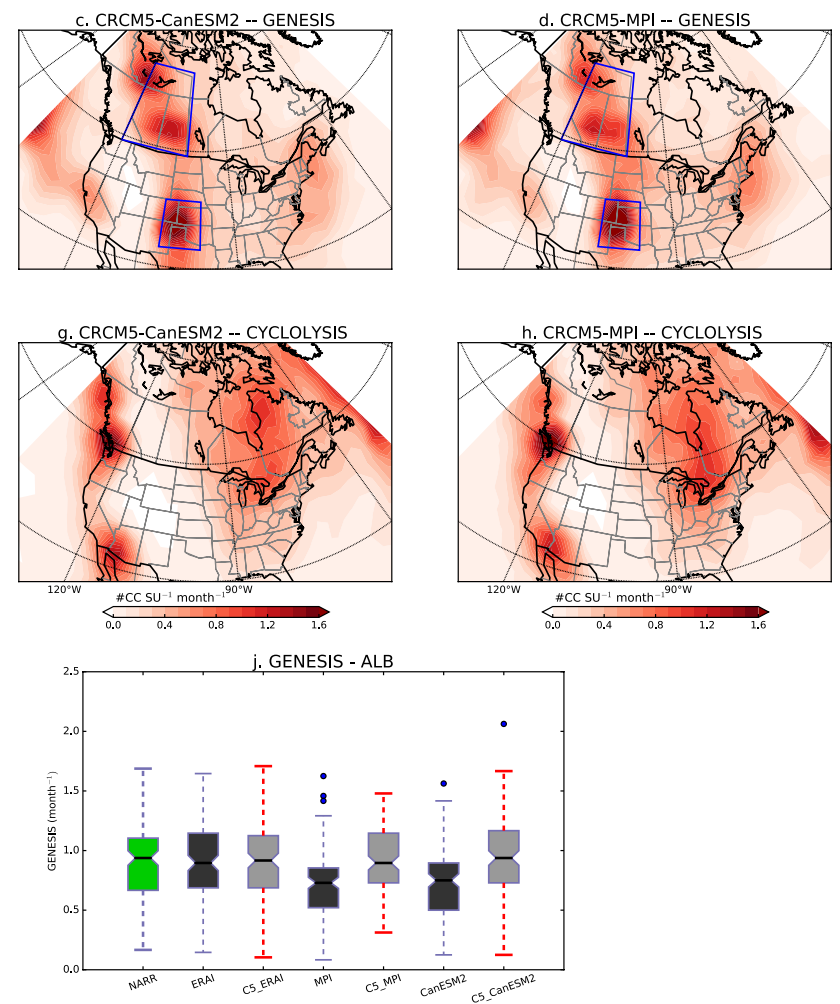

responding CRCM5 simulations are in gray with red whiskers. Boxplots show the min, max and the 3 quartiles (25th, 50th, 75th percentile) and also the outliers (in blue) that fall out of the interval [Q1-1.5 × IQR, Q3-1.5 × IQR] where Q1 and Q3 are the first and third quartiles and IQR the Inter Quartile Range. Note that C5 (i, j) corresponds to CRCM5 
and Alberta). Similarly, the CanESM2-driven simulation genesis (Fig. 6c) is improved over the continent and at the East Coast, as can be seen from the boxplots (Fig. 6i, j) where the median is shown to be very close to NARR one. A minor improvement is seen in MPI-ESMLR-driven CRCM5 (Fig. 6d), with the rate of cyclogenesis increasing towards the NARR rate over Alberta. It should be noted that, for both GCM-driven simulations, although significant improvement occurs for both mean (from $\sim 0.6$ to $\sim 1 \mathrm{CC} \mathrm{SU}^{-1}$ per month which is NARR value) and dispersion parameters over the Alberta region, the Colorado boxplots suggest that only the genesis (NDJFM) mean value is well captured by CRCM5, while dispersion is much more than observed. To sum up, despite this appreciable AV over continental areas, it appears that there still is room for improvement over the East Coast where CRCM5 tends to reproduce (or mimic) the patterns of GCMs, resulting in underestimation or spatial shift of genesis in comparison with NARR. Finally, the cyclolysis rate shows consistency among all regional simulations. Over the eastern sides of the Great Lakes and Hudson Bay, CRCM5 increases the cyclone dissipation frequency, which was underestimated by both GCMs. The same improvement can be seen over the southern California coast.
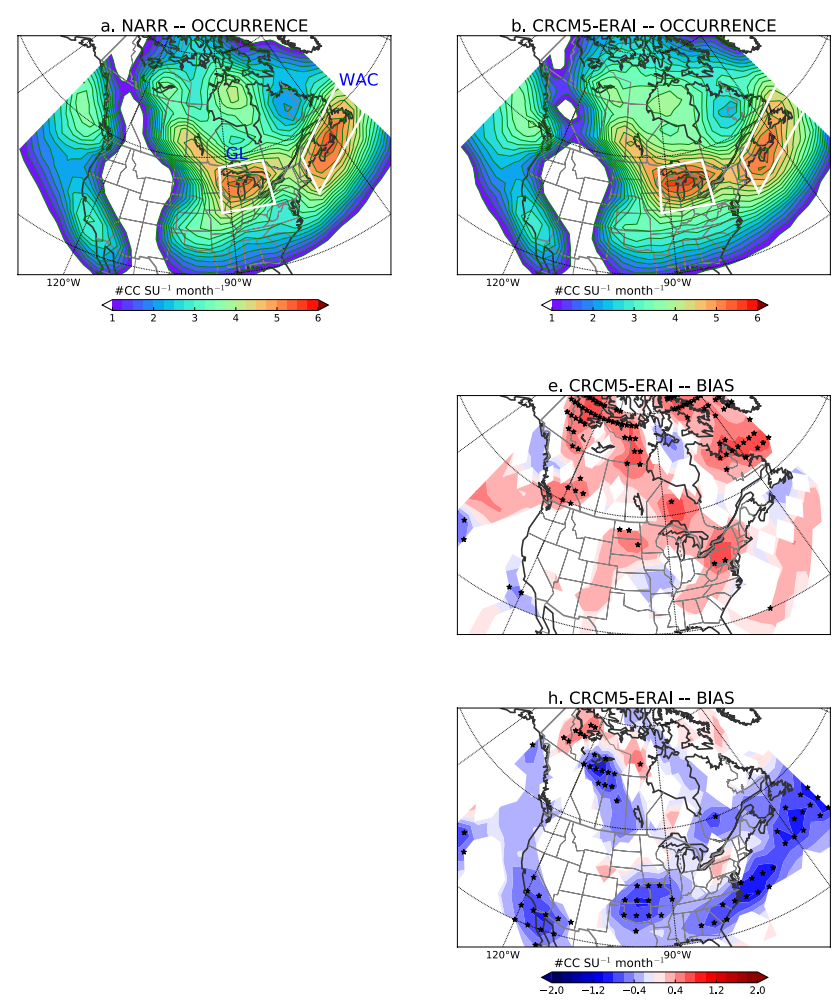
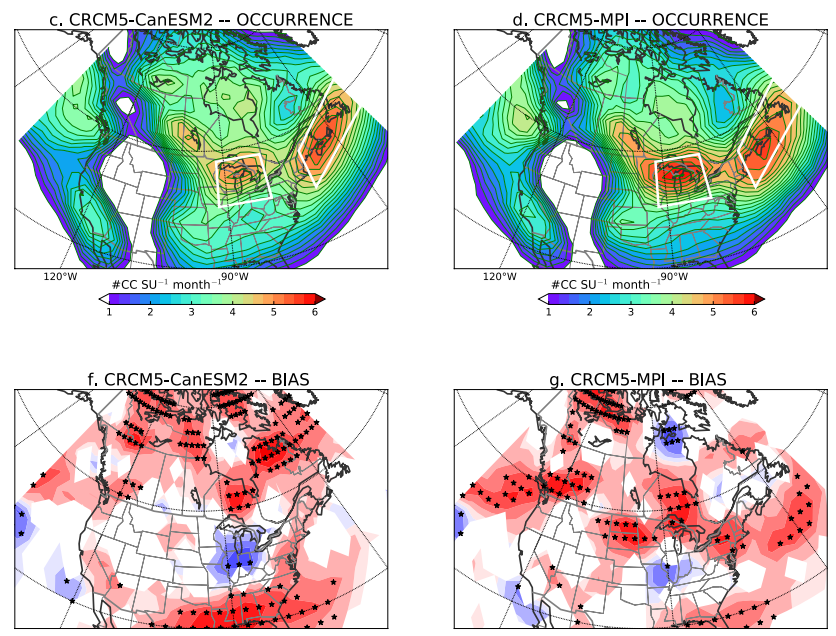

\subsubsection{Storm occurrence and intensity}

Figures 7 and 8 present storm occurrence and intensity (respectively) simulated by CRCM5 driven with the three different boundary conditions, together with the associated biases with respect to NARR and ERAI. In addition, to evaluate each variable distribution more accurately, boxplots of storm occurrence and intensity spatially averaged over the Great Lakes and the WAC are displayed in Fig. 9. Among the three versions of CRCM5, the ERAI-driven one clearly shows the lowest and mostly statistically non-significant biases for both occurrence (Fig. 7b, e) and intensity (Fig. 8b, e) parameters and for the whole of NA excluding arctic regions. In fact, the ERAI positive occurrence bias (discussed in Fig. 3b) is reduced by about a factor two over the continent, along the East Coast and over the Atlantic by the ERAI-driven CRCM5. On the other hand, Fig. $8 \mathrm{~b}$ suggests that, for the intensity parameter, both ERAI and CRCM5-ERAI biases are comparable (for instance over the Great Lakes) and are mostly not statistically significant.

GCM-driven simulations (Fig. 7c-f, h, i) display large and statistically significant occurrence biases, especially over the southeast of the USA and onto the Atlantic ocean when comparison is made with ERAI. Nevertheless, when the RCM is considered with respect to its driving GCM, Fig. 9a (for WAC occurrence) shows a

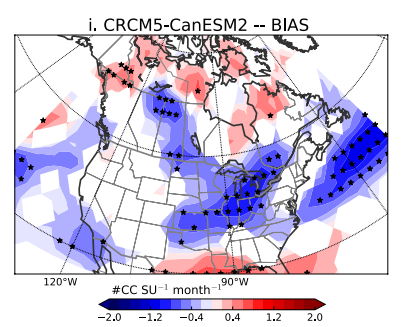

Fig. 7 Same as Fig. 3 but for CRCM5 simulations driven by ERAI (b, e and $\mathbf{h})$, CanESM2 (c, $\mathbf{f}$ and $\mathbf{i})$ and MPI-ESM-LR (d, $\mathbf{g}$ and $\mathbf{j})$ 

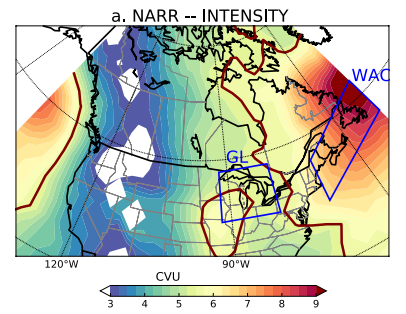
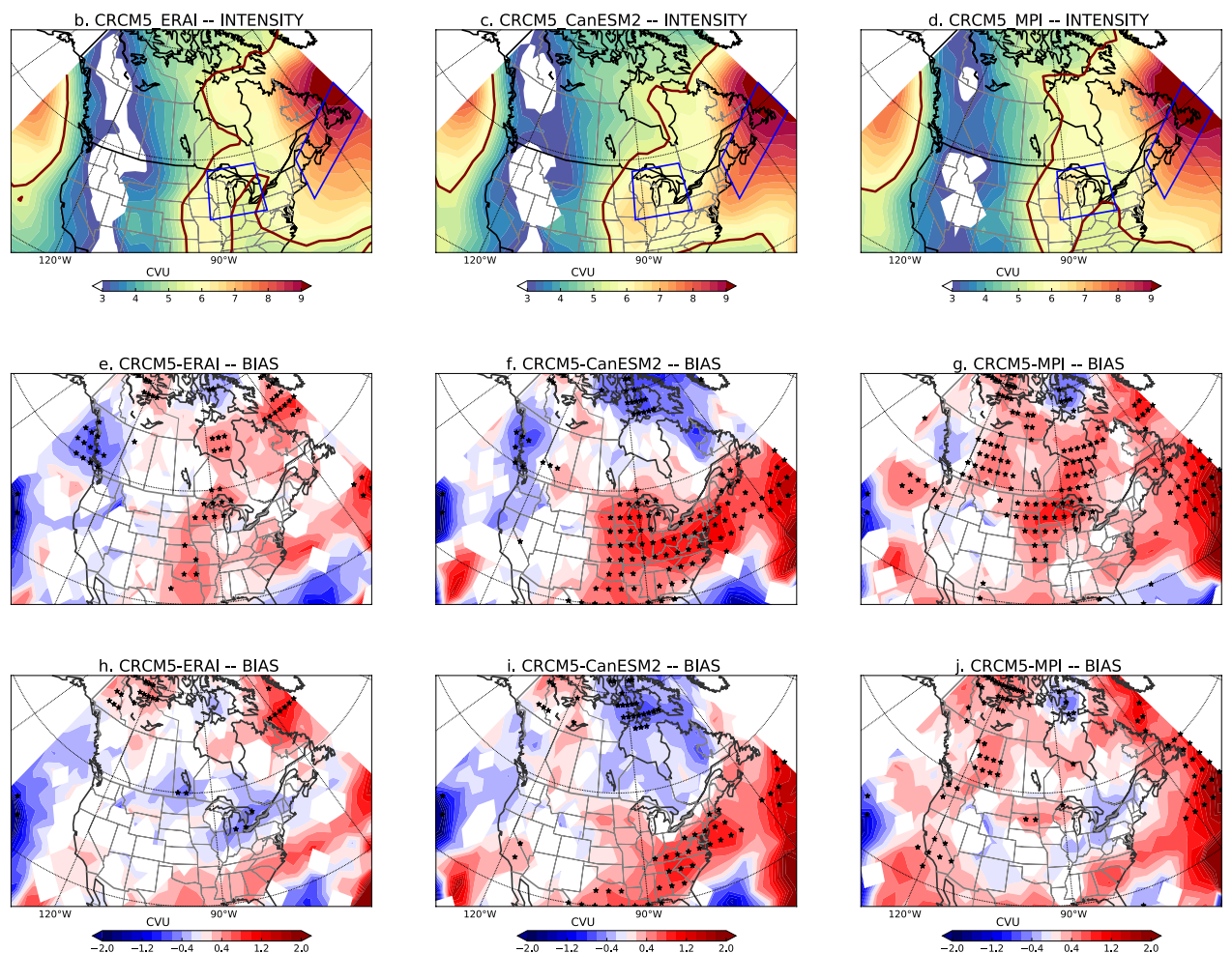

Fig. 8 Same as Fig. 4 but for CRCM5 simulations driven by ERAI (b, e and h), CanESM2 (c, f and i) and MPI-ESM-LR (d, $\mathbf{g}$ and $\mathbf{j})$
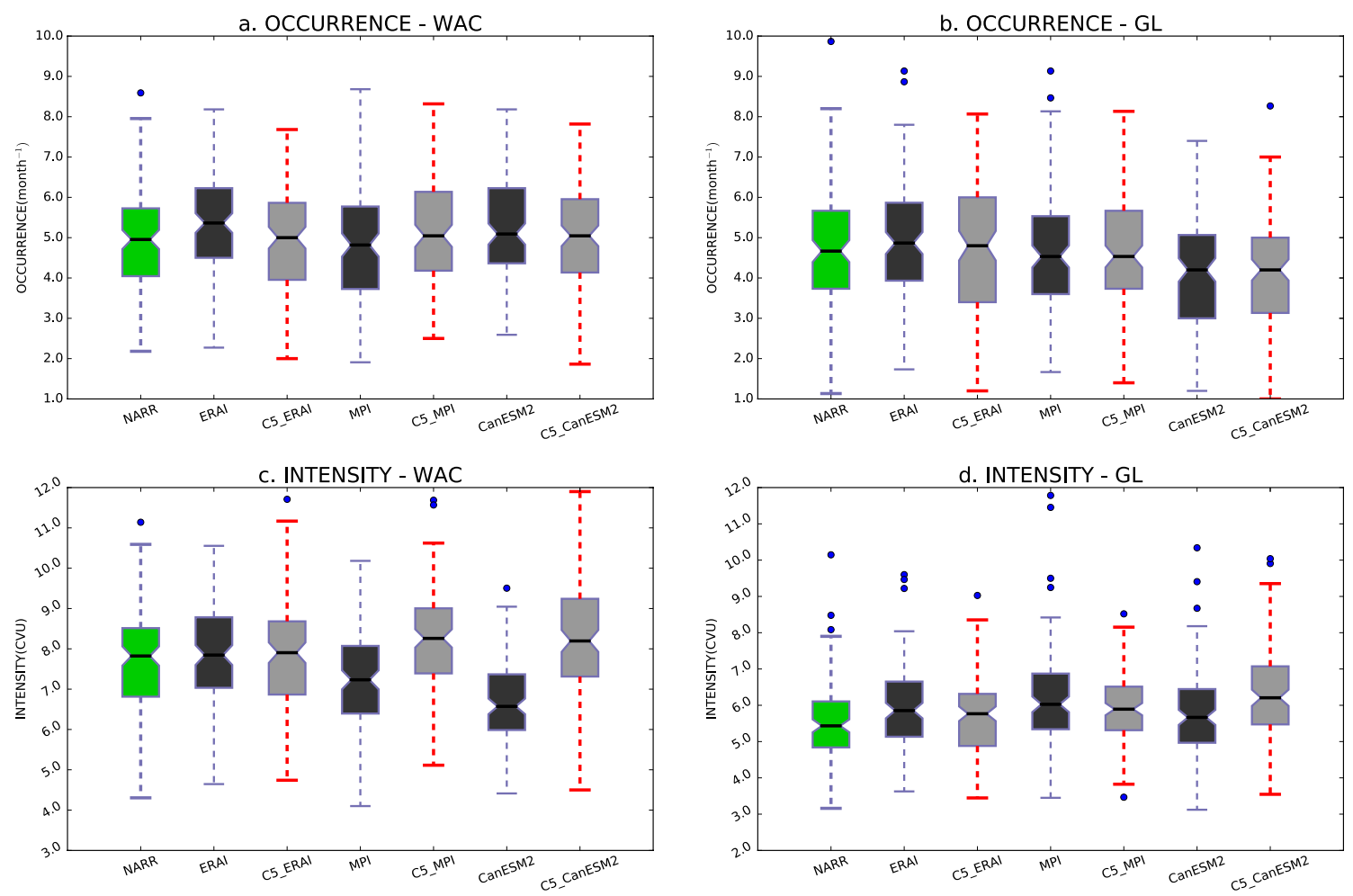

Fig. 9 Same as Fig. 6i, j but for monthly storm occurrence and intensity over the Western Atlantic Coast (WAC, a and c) and the Great Lakes (GL, b and d) 
slight improvement in terms of storm occurrence over the Western Atlantic coast, with, for instance, an interquartile range that is more compatible with NARR for the CRCM5 simulations. Conversely, over the Great Lakes (Fig. 9b), no clear difference appears with respect to GCM simulations.

Considering the EC mean intensity (Fig. 8), GCM negative bias is replaced by a positive bias along the majority of the East Coast in the CRCM5 runs (Figs. 8c, $\mathrm{d}, \mathrm{f}, \mathrm{g}, \mathrm{i}, \mathrm{j}, 9 \mathrm{c}$ ), which is potentially due to the exacerbating influences of positive biases (too warm) in the SSTs over the western Atlantic (Gulf Stream area) including over the Labrador Sea in the MPI runs, as suggested in Sect. 3.1.2. Finally, it appears that, near areas with marked relief, CRCM5 captures EC occurrence and intensity accurately and thus brings clearer AV than over the south east of North America and the North Atlantic.

\section{Temporal seriality of North America ECs}

This section analyzes the 'seriality' of storms based on their monthly count (occurrence) time series at model grid point scale. The dispersion parameter described in Sect. 2.2.2 is firstly assessed to diagnose CRCM5 (and driving model) skill in capturing some aspects of the intraseasonal (monthly) variability related to EC activity. Furthermore, regional storm occurrence and intensity distributions are assessed in order to determine the NA storminess regimes.
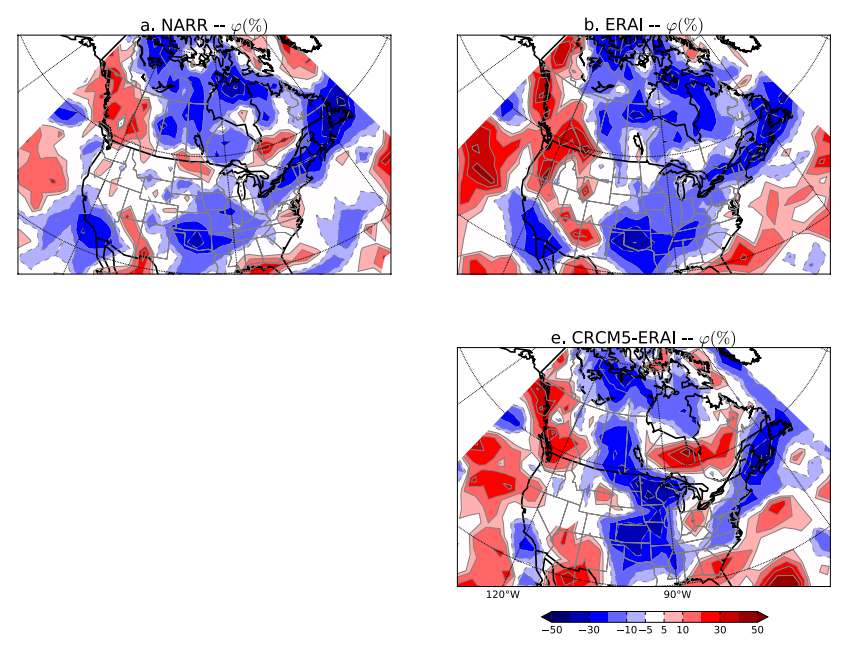

Fig. 10 25-year climatology of storm dispersion parameter $\varphi$ (in \%) computed from monthly storm occurrence at each grid point. NARR, ERAI, CanESM2 and MPI-ESM-LR realizations are displayed on the

\subsection{Storm dispersion maps}

Figure 10 displays the dispersion parameter $\varphi$ (in \%) in the same manner as in Pinto et al. (2013). Over the northern regions (Western and Northern Hudson Bay, and Canadian Arctic), there is decent consistency among all datasets, showing that storm occurrence is under-dispersive $(\varphi<0)$. In other words, it is a recurrent and regular phenomenon and so its monthly variability is rather weak over these regions, in agreement with Serreze (1995) and Stewart et al. (1995), who diagnosed frequent cyclogenesis activity (due to polar lows and associated blizzards) over the (Canadian and Alaskan) Arctic throughout the winter season.

Over the continental US, NARR (Fig. 10a) displays a southwest-northeast axis of storm regularity, in agreement with Vitolo et al. (2009) and Mailier et al. (2006), suggesting persistent storminess throughout the winter season. Such a regime originates from the eastern flank of the Colorado genesis region, passing successively across the Great Lakes, the St. Lawrence valley and the East Coast. This is also consistent with the quasi-permanent baroclinic energy generated by southern warm air masses (Gulf of Mexico and WAC) and the winter continental and/or polar cold air masses. On the other hand, close to the very continental cyclogenesis "points" (Alberta and Colorado as shown in Fig. 3a) and westward, storm occurrence is rather intermittent (clustered), partly reflecting the frequency at which upper tropospheric shortwave troughs cross and "emerge from" the Rocky Mountains. Despite significant differences in terms of seasonal mean occurrence, ERAI (Fig. 10b) seriality patterns are in good agreement with NARR over the majority of North America. However, noticeable differences can be seen over the Atlantic (along the Gulf Stream
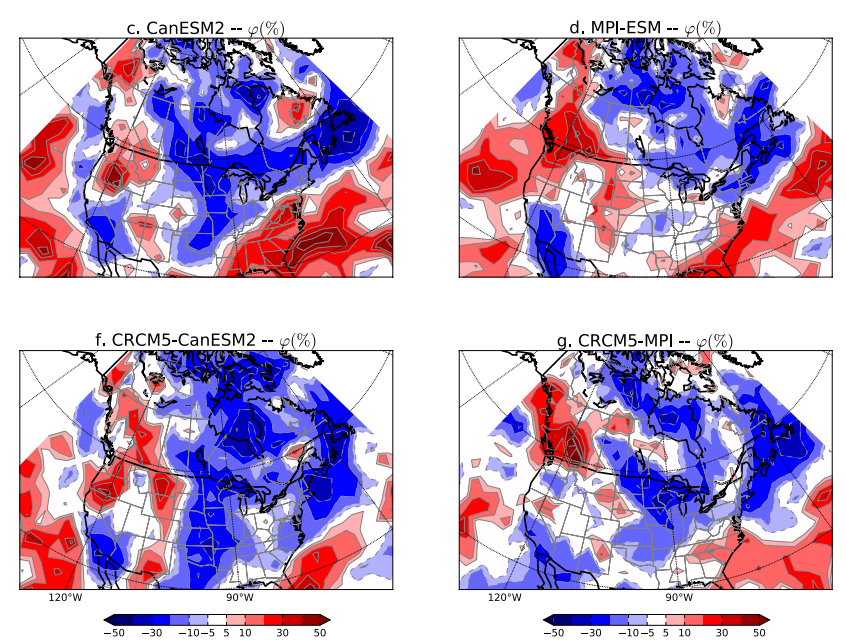

top panels and the respective CRCM5 driven conditions are displayed on the bottom panels 
area, see Fig. 5a) where ERAI storm occurrence is more intermittent (than in NARR data) but consistent with the results of Pinto et al. (2013). The CRCM5-ERAI (Fig. 10e) simulation shows comparable spatial patterns and magnitudes with respect to NARR, suggesting that the RCM is able to handle the intraseasonal variability of storm occurrence, except for the region between the northwestern Great Lakes and southern Alberta, where it gives more variability than in the observed persistent regime. Over the western topographic areas, like the other, previously analyzed parameters, the RCM tends to outperform ERAI.

Considering GCM simulations (Figs. 10c, 6d), the CanESM2 tends to outperform the MPI-ESM-LR model for most continental areas. The North Atlantic storm track entrance is relatively well defined in CanESM2, extending the regular regime from the East Colorado region to the Canadian Maritimes. On the other hand, the MPI-ESM-LR storm occurrence is either intermittent or neutral (total randomness i.e. Poisson process) over most of the continental USA, contrasting with the strong regularity found with NARR and ERAI (and equivalent downscaling results). In addition, the regular pattern in MPI-ESM-LR that occurs farther toward the east coast (New England States) is still not reproduced with the correct magnitude, shifting the observed maximum from the Newfoundland area to the west, along the land-sea shorelines of the Gulf of St. Lawrence. The GCM-driven CRCM5 simulations (Fig. 10f, g) seem to be very constrained by their respective GCMs, consistently with the idea that (RCM) storminess and particularly its seriality (that can be viewed as the rate at which the RCM is excited) are driven by large-scale influences inherited from the (potentially biased) boundary conditions. Nevertheless, some details seem to be improved in the RCM simulation, in particular over the area with marked relief (more compatible spatial structure) and over the Atlantic, where both GCMs tend to overestimate the dispersion, creating excessively intermittent patterns from the southeastern USA and along the Gulf Stream area. Finally, it is interesting to note that CRCM5 is able to correct the MPI-ESM-LR dispersion over the continent, extending the regular pattern inland (e.g. east of Colorado).

\subsection{Distribution of EC characteristics}

The previous subsection has given a broad view of the storminess regime within the winter season, showing some regions with persistent activity and others with rather clustered activity. In the following, regional domain analysis of the monthly occurrence and intensity will contribute more insight into the characteristics of each regime.

Figure 11 displays kernel density estimates (KDE, left $\mathrm{y}$-axis) and the resulting cumulative density functions (CDF, right $\mathrm{y}$-axis) of monthly time series of $\mathrm{EC}$ occurrence (top panels) and intensity (bottom panels) retrieved from the 4 regions (shown in Fig. 1). For each box, all grid point values are used to build the boxplots, thus allowing both spatial and temporal dispersions to be included.

Over the WAC area (Fig. 11a), NARR occurrences range from about 0 to 12 storms per month, with a statistical median and mode of around 4 storms per month. The regular regime is materialized by the compact shape of the distribution, implying that relatively small dispersion occurs around the mean occurrence value $(\sim 5$ storms per month). ERAI distribution is shifted toward the righthand side, suggesting more presence of months with higher storm occurrence values, as suggested by the larger median (5 storms in ERAI instead of 4 in NARR). This distribution shift also suggests that ERAI positive occurrence bias over the coastal area (Fig. 3) may be related to a larger than observed contribution of very active regimes, with an occurrence range of about $6-9$ storms per month during the winter season. It is shown that ERAI-driven CRCM5 significantly reduces the shift of the pattern, leading to better agreement in terms of median and modes with respect to NARR. The intensity KDE shows that, in this WAC regular regime, storm intensity has a median value of $\sim 7 \mathrm{CVU}$ and is pretty well reproduced by ERAI and CRCM5 compared with the NARR distribution. It is worth adding that, even for extreme events (12-14 CVU), the RCM still behaves very well. On the other hand, MPI-ESM-LR and CanESM2 models show fair skill in reproducing the observed shape of the occurrence distribution in a manner very comparable to ERAI. Interestingly, it is shown that, as with ERAI, the regionally downscaled simulations can still bring some improvement, especially for a weaker occurrence regime. Finally, serious discrepancies arise in the EC intensity distribution of the CanESM2, in which a weaker storm regime dominates the distribution. The correction brought by CRCM5 is very meaningful even though there is an overestimation of higher intensity storms in return. This too-intense storm category, captured by both GCM-driven CRCM5 runs, could be linked with the SST warm biases inherited from the GCM simulations.

A point of agreement among all data sets is that storm occurrence frequencies are generally distributed over smaller values (and with weaker intensity) over the Great Lakes region (Fig. 11b) than over the WAC region. In addition, the compact shape of the GL occurrence distribution is still noticeable for all datasets, although with a less regular regime (see Fig. 10). ERAI and its driven CRCM5 perform very well with respect to NARR, exhibiting a regular storminess regime centered on 4 storms per month. On the other hand, although a slight improvement can be seen from CRCM5, storm intensity appears biased toward higher values in both ERAI and CRCM5 in comparison 


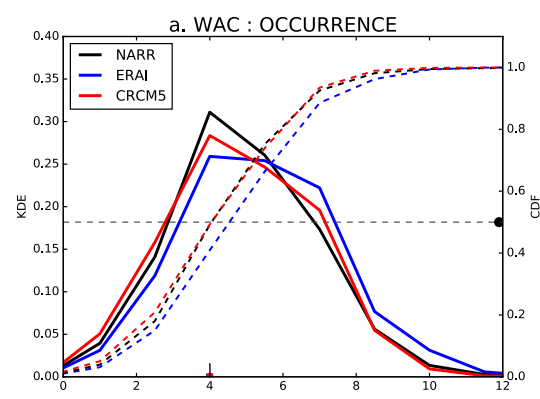

(A) Western Atlantic Coast
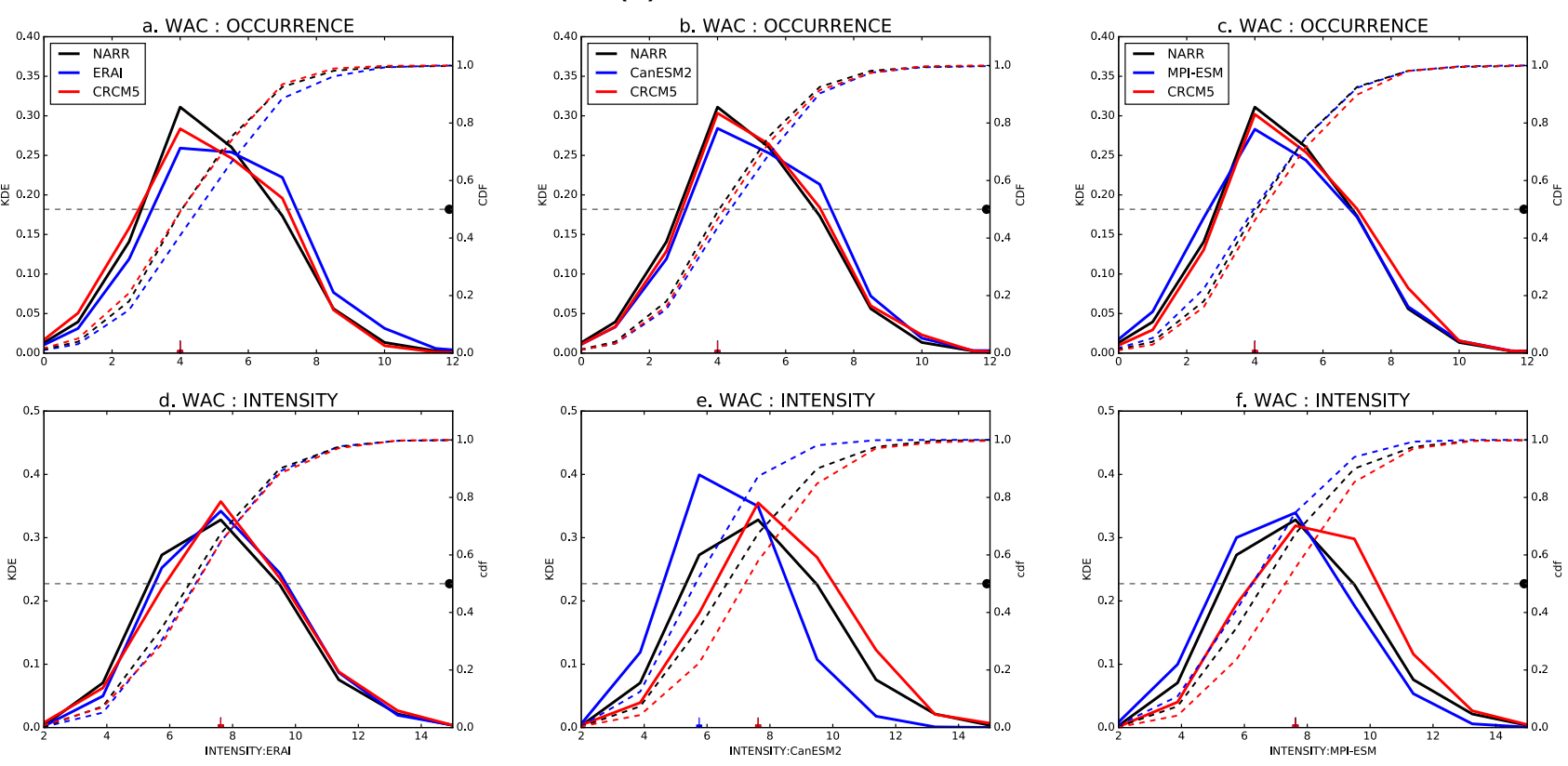

(B) GreatLakes
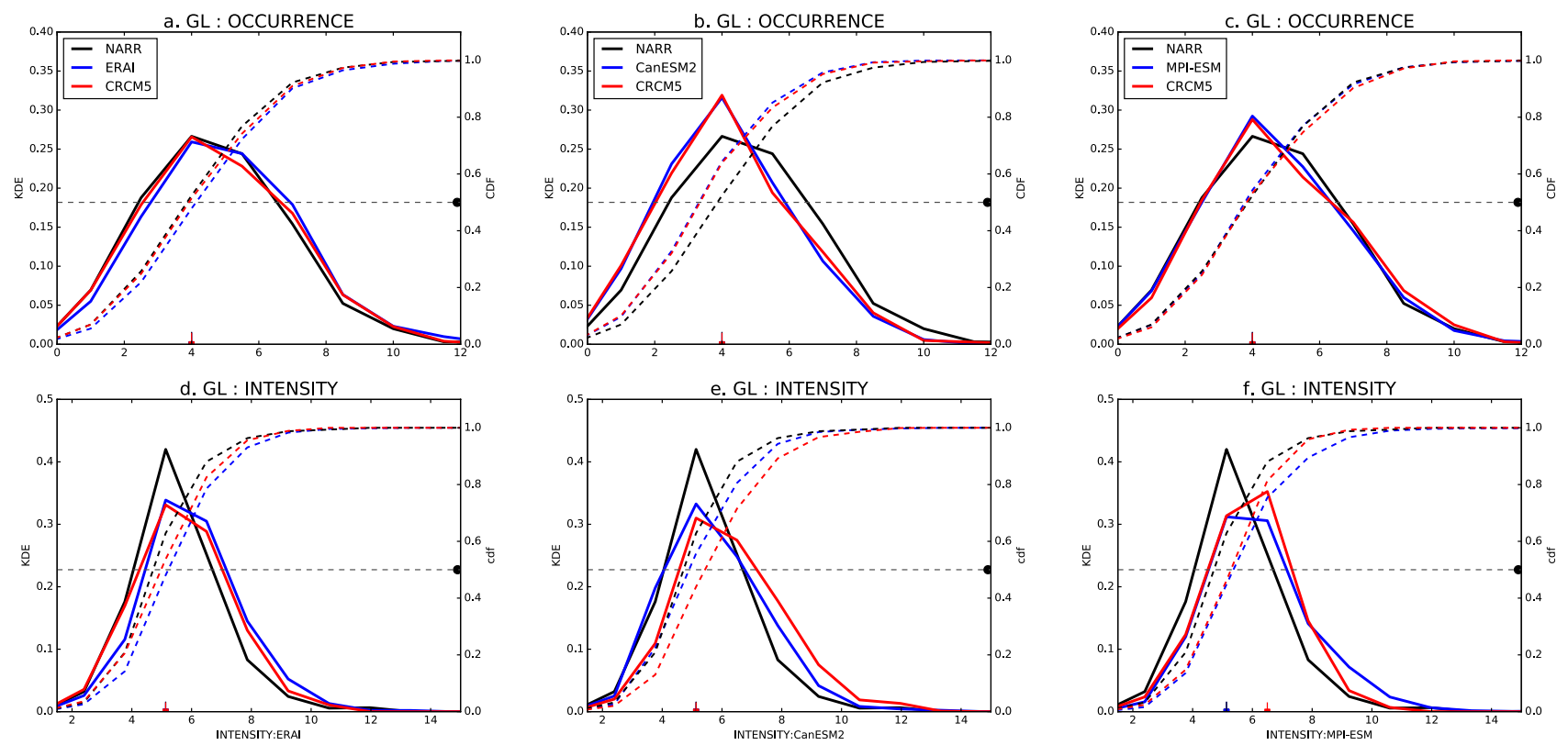

Fig. 11 Kernel density estimates (KDE) of 25-year NDJFM monthly storm parameters computed over the 4 regions delimited by blue boxes in Fig. 1: a Western Atlantic coast, b Great Lakes, c Colorado and d Alberta. Each column represents a given simulation monthly storm occurrence (storm month ${ }^{-1}$, top row) and intensity (CVU, bottom row) distributions. The cumulative density function computed from the integral of $\mathrm{KDE}$ is shown in dashed curves and the horizontal black-dashed line indicates the CDF 0.5 value. On each plot, NARR is in black, the driving data in blue and the corresponding CRCM5 run in red. On the $x$-axis, markers show the statistical mode values for each data set

regional model seems to mimic its driving dataset, leading to a systematic overestimation of the occurrence of intense storms. This is a counterintuitive outcome since one would expect the RCM to be able to capture the Great Lakes effects and therefore not to mimic the large-scale model. with NARR. This means that these two models would tend to show a storm regime that is more intense than observed (between 7 and $10 \mathrm{CVU}$ ). More generally, over this region, it is difficult to conclude on whether CRCM5 improves both parameters in comparison with its driving data. In fact, the 


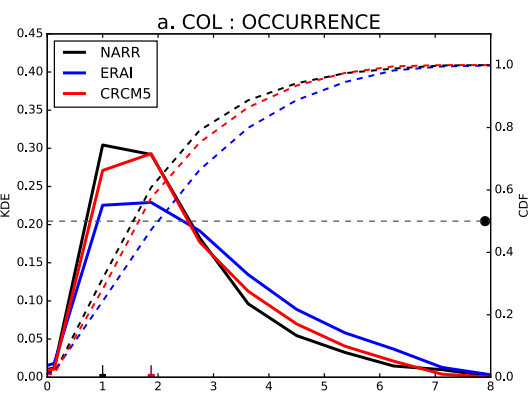

(C) Colorado
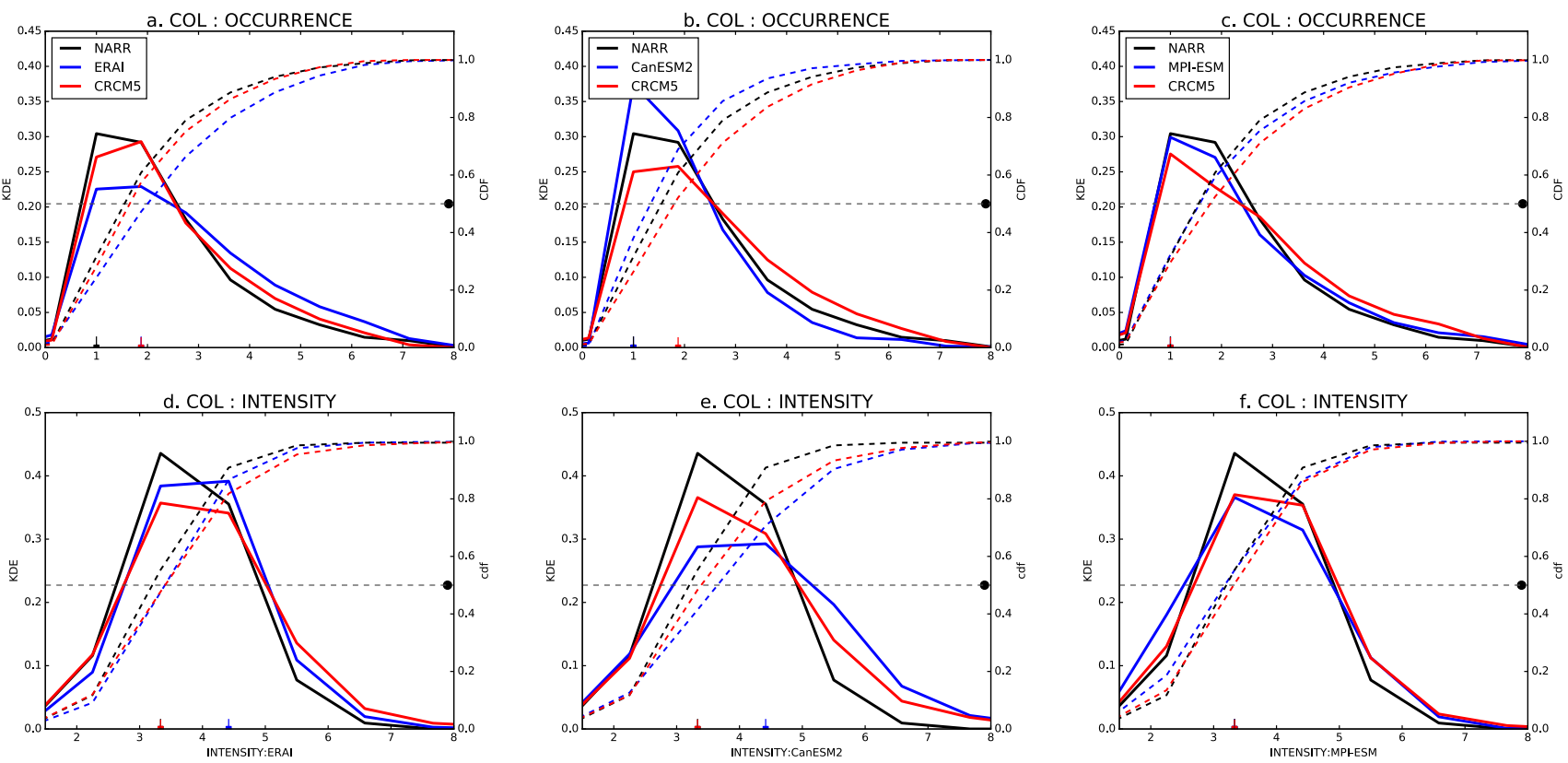

(D) Alberta
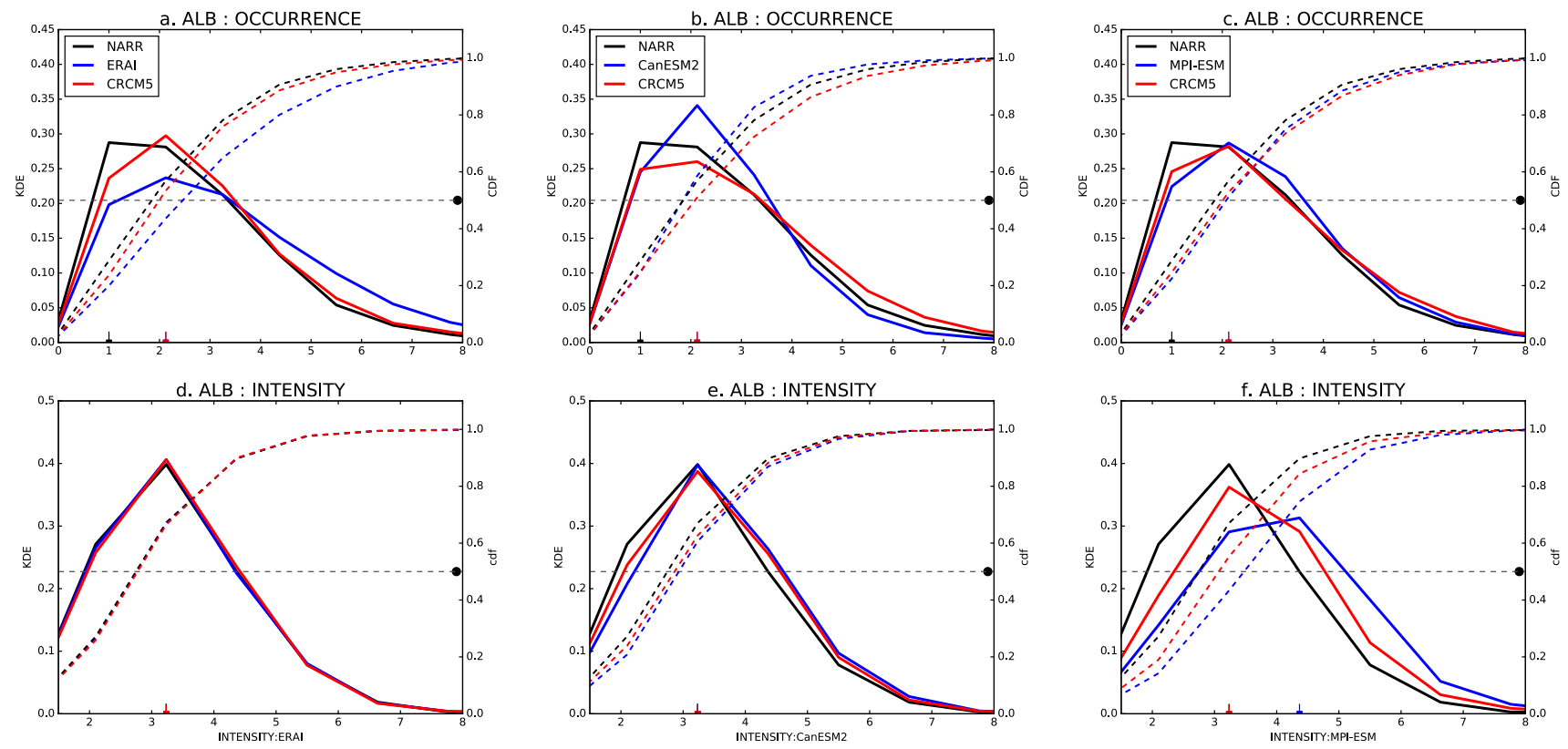

Fig. 11 (continued)

The analysis reveals that, because of the smaller footprint of the GL effects (in opposition to the WAC), more resolution and certainly more refinement in the surface coupling system are needed to "free" the RCM from its driver.

Finally, over the continental cyclogenesis regions (COL and ALB, Fig. 11c, d, respectively), the intermittent regime already seen in Fig. 10 is well materialized with (right-hand) long-tailed distributions for all datasets.
EC occurrence is a very dispersive process, occurring with relatively weak mean value ( 2 storms per month) but marked by regimes with high occurrences ( $4-8$ storms per month). Figure $11 \mathrm{c}, \mathrm{d}$ show that the regional model tends to improve the spatio-temporal distribution of storm occurrence and intensity with respect to ERAI and CanESM2 (and MPI-ESM-LR for the EC intensity over the Alberta area only, see Fig. 11e). On the other hand, the RCM skill 
remains comparable with that of MPI-ESM-LR for the occurrence over the two regions.

Hence, in general for the majority of regions (except the Great Lakes area) and EC characteristics, the CRCM5 simulations tend to improve the occurrence and intensity of storms from their reanalysis and GCM-driven runs. Over the Great Lakes area, the RCM is more dependent on its driven conditions and the AV is less obvious or tenuous, i.e. the regional scale influences and the value added to EC features are less marked than over the other continental and maritime areas where both high-resolution topographic and land/sea boundary conditions can emerge.

\section{Conclusion and future work}

The paper has evaluated the value added by the Canadian Regional Climate Model-version 5 (CRCM5) when simulating northern American ECs as one of the major components influencing mid-latitude weather and climate states. Because complex physics at different time and space scales is involved, assessing model performance with respect to EC genesis and development requires detailed analyses, and the current paper has attempted to disentangle some of them. Thus, both seasonal characteristics and intraseasonal variability have been assessed and the main findings are:

- The choice of a reference dataset for model comparison is an important factor for storm tracking analysis. The present analysis-in agreement with Hodges et al. (2011) - found that ERAI mostly overestimates storm genesis and occurrence (and, to a lesser extent, intensity) over North America with respect to the regional reanalysis. Because of these differences among reanalyses, some results from previous studies assessing the performance of (global and regional) climate models need to be taken with caution, especially when regionalscale processes are implicated and interact strongly with large-scale flow and ECs over North America. More fundamentally, there is a need to reach a consensus on the notion of reference data. This consensus may vary depending on the applications or the diagnostic variables considered (e.g. thermodynamical or diabatic forcings, storm tracking, etc.), and the region of interest.

- The two GCM simulations capture the overall picture of storm activity over North America, confirming results from previous studies (Zappa et al. 2013). However, systematic large biases appear over the western marked relief area where the GCM's relatively lower height of mountains leads to an overestimate of cyclone occurrence and intensity. This is thought to be a minor (or solvable) issue since GCM resolution will continue to increase. Near water masses (Hudson Bay, Great Lakes and East Coast), it is clear that both parameters are underestimated. Comparison with ERAI suggests even more amplified biases over the East Coast in line with previous results. It is also surprising that, despite strong SST warm biases, GCMs-especially CanESM2—still underestimate storm genesis and intensity over the East Coast. Interestingly, Zappa et al. (2013) have shown that guiding GCMs with "observed" SST does not bring significant improvement. This lack of sensitivity over the western warm boundary current may be due to: (1) the limitation of horizontal resolution in the GCMs since it is shown that SST gradients (as well as amplitudes) need to be well resolved in both the time and space dimensions to correctly impact EC dynamics and (2) physical parametrization of surface latent and sensible heat fluxes (e.g., Bourassa et al. 2013). This last point is supported by the fact that CRCM5, even inheriting from the same (spatial and temporal resolution) SSTs, was at least able to simulate some added value over this area for both occurrence and intensity characteristics and also in terms of persistence or regularity with respect to boundary conditions. It is recalled that CRCM5 uses a weather forecast model physics, the Environment Canada Global Environmental Multiscale (GEM) forecast model (Côté et al. 1998a, b). This means that this regional model uses an improved physical package compared to GCM-scale model to resolve short term processes and high frequency turbulent fluxes. Indeed, the planetary boundary layer parameterization in the GEM forecast model has been regularly improved and modified during the course of time (see a review used in the CRCM5 in Martynov et al. 2013), for example in introducing turbulent hysteresis to improve the representation of the synoptic scales (i.e. ECs in winter; see Zadra et al. 2012).

- CRCM5 driven by ERAI shows remarkable skill over the whole NA domain, outperforming ERAI, particularly near mountains (Midwest Rockies). Regional boxplots have shown that cyclone genesis, intensity and occurrence are remarkably improved with respect to NARR, showing more accurate statistics (median and quartiles) than ERAI ones. On the other hand, the dynamically downscaled simulations have revealed, with respect to GCMs, that the notion of added value is quite complex to assess since it involves, at least, resolution and physics issues. For instance, the continental cyclogenesis and lysis areas (Colorado and Alberta) appeared well captured in CRCM5 while the Great Lakes region occurrence and intensity remained comparable to those provided by GCMs. However, it seems that, over the East Coast, in contrast with GCMs, SST biases impact CRCM5 performance in a more comprehensive way: positive SST biases would lead to 
stronger cyclones (consistently with Booth et al. 2012). And, more generally, the sensitivity of any RCM to changes (or biases) in surface conditions is more pronounced than in any of the coarse scale models, due to the improved resolution of the RCM, which will bring out amplified effects of surface and low level diabatic fluxes not resolved at the scale of a GCM. Nevertheless, quite a substantial improvement has been revealed with respect to storm occurrence in the CRCM5 simulations over the WAC area, with also noticeable agreement for storm intensity in the case of CanESM2 driving simulation.

- In an attempt to characterize the storminess regimes within the winter season and thus the intra-seasonal variability, the dispersion parameters and monthly occurrence (and intensity) distributions were assessed. It is shown with NARR (and generally by all datasets) that the eastern part of NA is subject to a persistent (regular) regime while that of the western part is more intermittent, characterized by temporally clustered storms. Although it seems obvious that the RCM seriality is somehow imposed by boundary conditions, the dispersion parameter shows that substantial improvement is brought by using CRCM5 over the continental cyclogenesis regions and on to the Atlantic. The time series of monthly occurrence and intensity density function analysis over the four domains also shows that, in general, the RCM is more accurate in refining the storm activity regimes (statistical medians, mean, modes and extreme event tails for occurrence and intensity).

To sum up, the paper has confirmed the reasonable idea that an RCM is able to reproduce and improve our capacity to simulate EC characteristics in a climate perspective. That has been demonstrated by the skill of ERAI-driven simulations, which handle extratropical storm characteristics (genesis, occurrence, intensity, dispersion) fairly well (and partly even better than ERAI). On the other hand, the added value that an RCM brings to GCMs is tangible firstly through resolution effects and then through improved physics. In particular over the East Coast, despite largely biased boundary conditions (in the CanESM2 driven version) and despite the fact that those bias effects naturally tend to be exacerbated at higher resolution (e.g. RCM resolution), the CRCM5 has revealed serious potential, mainly for improving storm intensity distribution. However, further work is needed to fully evaluate the whole potential added value for the CRCM5 versus coarse scale boundary conditions, and in particular the effect of different time resolution simulations used here in the storm tracking ( 3 hourly for RCM and NARR while 6 hourly for the rest) on sensitive EC features (ex. storm speed and phase of rapid intensification during explosive developments over the eastern NA coast). Furthermore, all data were spatially re-gridded to a common $100 \mathrm{~km}$ horizontal grid resolution, to facilitate the comparison with an intermediate resolution between the RCM $(\sim 50 \mathrm{~km})$ and GCM $(\sim 200 \mathrm{~km})$. As we were mainly interested to evaluate the added value of RCM and our focus is on winter synoptic scale storms, i.e. in general with a size more often higher than $1500 \mathrm{~km}$, this interpolation was not detrimental for RCM, but can potentially induce some artefact in GCM scale values (not necessarily problematic as large scale systems are concerned). Nevertheless, this needs to be evaluated in future works, i.e. to analyze to what extent those space and time differences may impact the results and to be able to generalize our conclusion. Finally, considering the inherent sensitivity of EC characteristics to the surface conditions in winter, i.e. SST gradients and location of sea-ice margin over the eastern coast of North America, further works need to be done using CRCM5 simulations with unbiased SSTs (e.g., Hernández-Díaz et al. 2016). Also, different RCMs need to be used for an in-depth evaluation of the uncertainties brought by the downscaling model and to develop more robust EC regional scale information over the North America region, by using various combinations of GCMs and greenhouse gas emission scenarios (GES). This work is already underway and is using different CORDEX runs with two GES to generate climate change information, the links between changes in storm track occurrence and intensity, and the modification in the precipitation and the wind regime over the coming decades. This is of particular importance as ECs over eastern North America have the most pronounced effects on intense precipitation, including heavy snowfall events, and coastal flood risks occurring from the fall to spring months.

Acknowledgements The authors acknowledge the financial support of the Canadian Network for Regional Climate and Weather Processes (CNRCWP-http://www.cnrcwp.uqam.ca/), funded by the Natural Sciences and Engineering Research Council of Canada (NERSCCRSNG-http://www.nserc-crsng.gc.ca/index_eng.asp). We are also grateful to the Centre pour l'Étude et la Simulation du Climat à l'Échelle Régionale (ESCER) of the Université du Québec à Montréal (UQAM) for providing the outputs of CRCM5 simulations used in our study. We specifically thank Ms Katja Winger and Prof. Laxmi Sushama at UQAM, who provided information and output files from CRCM5. We would also like to thank the CCCma/ECCC, ECMWF, NCEP and the Max-Planck Institute for providing output files for CanESM2, ERAI, NARR and MPI-ESM-LR, respectively. Finally, the authors sincerely thank the two anonymous reviewers whose contributions have helped to improve the results and the structure of the paper.

Open Access This article is distributed under the terms of the Creative Commons Attribution 4.0 International License (http:// creativecommons.org/licenses/by/4.0/), which permits unrestricted use, distribution, and reproduction in any medium, provided you give appropriate credit to the original author(s) and the source, provide a 
link to the Creative Commons license, and indicate if changes were made.

\section{References}

Ahrens CD (2009) Meteorology today: an introduction to weather, climate, and the environment, Brooks/Cole Edition (9th edn), Brooks Cole, Belmont, p 355

Allen JT, Pezza AB, Black MT (2010) Explosive cyclogenesis: a global climatology comparing multiple reanalyses. J Climate 23(24):6468-6484

Arora VK, Coauthors (2011) Carbon emission limits required to satisfy future representative concentration pathways of greenhouse gases. Geophys Res Lett 38:L05805. doi:10.1029/2010GL046270

Bengtsson L, Hodges KI, Roeckner E (2006) Storm tracks and climate change. J Climate 19:3518-3543

Blender R, Schubert M (2000) Cyclone tracking in different spatial and temporal resolutions. Mon Weather Rev 128:377-384

Bluestein HB, (1993) Synoptic-dynamic meteorology in midlatitudes. In: Observations and theory of weather systems, Vol. II, Oxford University, Oxford, p 594

Booth JF, Thompson L, Patoux J, Kelly KA (2012) Sensitivity of midlatitude storm intensification to perturbations in the sea surface temperature near the Gulf Stream. Mon Weather Rev 140:1241-1256

Bourassa MA et al (2013) High-latitude ocean and sea ice surface fluxes: challenges for climate research. Bull Amer Meteor Soc 94:403-423. doi:10.1175/BAMS-D-11-00244.1

Branscome LE, Gutowski WJ Jr, Stewart DA (1989) Effect of surface fluxes on the nonlinear development of baroclinic waves. $J$ Atmos Sci 46:460-475

Brayshaw DJ, Hoskins BJ, Blackburn M (2009) The basic ingredients of the North Atlantic storm track. Part I: Land-sea contrast and orography. J Atmos Sci 66:2429-2558

Chang EK, Lee S, Swanson KL (2002) Storm track dynamics. J Climate 15(16):2163-2183

Colle BA, Zhang Z, Lombardo K, Liu P, Chang E, Zhang M (2013) Historical evaluation and future prediction in Eastern North America and western Atlantic extratropical cyclones in the CMIP5 models during the cool season. J. Climate. 2013; 26:6882-6903

Colle BA, Booth JF, Chang EKM (2015) A review of historical and future changes of extratropical cyclones and associated impacts along the US East Coast. Curr Clim Change Rep 1:125-143. doi:10.1007/s40641-015-0013-7

Côté J, Desmarais J-G, Gravel S, Méthot A, Patoine A, Roch M, Staniforth A (1998a) The operational CMC-MRB global environmental multiscale (GEM) model. Part II: results. Mon Weather Rev 126:1397-1418

Côté J, Gravel S, Méthot A, Patoine A, Roch M, Staniforth A (1998b) The operational CMC-MRB global environmental multiscale (GEM) model. Part I: Design considerations and formulations. Mon Weather Rev 126:1373-1395

Côté H, Grise KM, Son S-W, de Elía R, Frigon A (2015) Challenges of tracking extratropical cyclones in regional climate models, Clim Dyn 44(11):3101-3109

Dee DP et al. (2011) The ERA-Interim reanalysis: configuration and performance of the data assimilation system. Q J R Meteorol Soc 137:553-597. doi:10.1002/qj.828

Di Luca A, de Elía R, Laprise R (2015) Challenges in the quest for added value of regional climate dynamical downscaling. Curr Clim Change Rep 1(1):10-21. doi:10.1007/s40641-015-0003-9
Eichler TP, Gottschalck JA (2013) Comparison of southern Hemisphere cyclone track climatology and interannual variability in coarse gridded reanalysis datasets. Adv Meteorol, p 1-16

Gachon P, Saucier FJ (2003) La modélisation du climat dans les mers intérieures du Canada : Baie d'Hudson et Golfe du Saint-Laurent. Nat Can 127(2):117-122

Gachon P, Saucier FJ, Laprise R (2001) Atmosphere-ocean-ice interaction processes in the Gulf of St. Lawrence: numerical study with a coupled model. In: Sixth Conference on Polar Meteorology and Oceanography and 11th Conference on Interaction of the Sea and Atmosphere. AMS (American Meteorological Society) Meeting, San Diego (14-18 May 2001. Abstract volume, $\mathrm{J} 1.18, \mathrm{~J} 37-\mathrm{J} 40$ (B))

Gachon P, Laprise R, Zwack P, Saucier FJ (2003) The effects of interactions between surface forcings in the development of a modelsimulated polar low in Hudson Bay. Tellus A 55(1):61-87

Giorgi F, Jones C, Asrar GR (2009) Addressing climate information needs at the regional level: the CORDEX framework. World Meteorol Org Bull 58:175

Goyette S, Brasseur O, Beniston M (2003) Application of a new wind gust parameterization: Multiscale case studies performed with the Canadian regional climate model. J Geophys Res 108(D13):4374. doi:10.1029/2002JD002646

Giorgetta et al (2013) Climate and carbon cycle changes from 1850 to 2100 in MPI-ESM-LR simulations for the coupled model intercomparison project phase 5. J Adv Model Earth Syst 5:572-597. doi:10.1002/jame.20038

Grise KM, Son S-W, Gyakum JR (2013) Intraseasonal and interannual variability in North American storm tracks and its relationship to equatorial Pacific variability. Mon Weather Rev 141:3610-3625

He J, Zhang M, Lin W, Colle B, Vogelmann A (2013) Simulations of a mid-latitude cyclone over the southern Great Plains using the WRF nested within the CESM. J Adv Mod Earth Syst 5:611-622

Hernández-Díaz L, Laprise R, Nikiéma O, Winger K. (2016) 3-Step dynamical downscaling with empirical correction of sea-surface conditions: application to a CORDEX Africa simulation. Clim Dyn. doi:10.1007/s00382-016-3201-9

Hirsch ME, DeGaetano AT, Colucci SJ (2001) An East Coast winter storm climatology. J Clim 14:882-899

Hodges KI, Lee RW, Bengtsson L (2011) A comparison of extratropical cyclones in recent reanalyses ERA-Interim, NASA MERRA, NCEP CFSR, and JRA-25. J Clim 24:4888-4906

Hoskins BJ, Hodges KI (2002) New perspectives on the Northern Hemisphere winter storm tracks. J Atmos Sci 59:1041-1061

Hoskins BJ, Valdez P (1990) On the existence of storm-tracks. J Atmos Sci 47:1854-1864

IPCC (2013), Climate change 2013: the physical science basis. In: Stocker TF, Qin D, Plattner G-K, Tignor M, Allen SK, Boschung J, Nauels A, Xia Y, Bex V, Midgley PM (eds) Contribution of working group I to the fifth assessment report of the intergovernmental panel on climate change. Cambridge University, Cambridge, p 1535

Jung T, Gulev SK, Rudeva I, Soloviov V (2006) Sensitivity of extratropical cyclone characteristics to horizontal resolution in the ECMWF model. Q J R Meteorol Soc 132:1839-1857

Kumar A, Zhang L, Wang W (2013) Sea surface temperature-precipitation relationship in different reanalyses. Mon Weather Rev 141:1118-1123. doi:10.1175/MWR-D-12-00214.1

Lackmann, G. (2011) Midlatitude synoptic meteorology: dynamics, analysis and forecasting, American Meteorological Society, Massachusetts 345

Lambert SJ, Fyfe JC (2006) Changes in winter cyclone frequencies and strengths simulated in enhanced greenhouse warming experiments: results from the models participating in the IPCC diagnostic exercise. Clim Dyn 26:713-728 
Laprise R (2008) Regional climate modelling. J Comp Phys, 227(Special issue on "Predicting weather, climate and extreme events"):3641-3666, doi:10.1016/j.jcp.2006.10.024.

Laprise R, Caya D, Frigon A, Paquin D (2003) Current and perturbed climate as simulated by the second-generation Canadian Regional Climate Model (CRCM-II) over northwestern North America. Clim Dyn 21:405-421

Liberato ML (2014) The 19 January 2013 windstorm over the North Atlantic: large-scale dynamics and impacts on Iberia. Weather Clim Extremes 5:16-28

Long Z, Perrir W, Gyakum J, Laprise R, Caya D (2009) Scenario changes in the climatology of winter midlatitude cyclone activity over Eastern North America and the northwest Atlantic. J Geophys Res 114:D12111

Mailier PJ, Stephenson DB, Ferro CAT, Hodges KI (2006) Serial clustering of extratropical cyclones. Mon Weather Rev 134:2224-2240

Marciano CG, Lackmann GM, Robinson WA (2015) Changes in U.S. East Coast cyclone dynamics with climate change. J Climate. doi:10.1175/JCLI-D-14-00418.1

Martynov A, Sushama L, Laprise R (2010) Simulation of temperate freezing lakes by one-dimensional lake models: performance assessment for interactive coupling with regional climate models. Boreal Environ Res 15(Special issue on lakes in atmospheric models):143-164 (issn 1797-2469)

Martynov A, Laprise R, Sushama L, Winger K, Šeparović L, Dugas B (2013) Reanalysis-driven climate simulation over CORDEX North America domain using the Canadian Regional Climate Model, version 5: Model performance evaluation. Clim Dyn 41(11-12):2973-3005

Mearns LO, Gutowski WJ, Jones R, Leung L-Y, McGinnis S, Nunes AMB, Qian Y (2009) A regional climate change assessment program for North America. Eos 90:311-312

Mesinger F, DiMego G, Kalnay E, Mitchell K, Shafran PC, Ebisuzaki W, Shi W (2006) North American Regional Reanalysis. Bull Am Meteorol Soc 87:343-360. doi:10.1175/BAMS-87-3-343

Murray RJ, Simmonds I (1991) A numerical scheme for tracking cyclone centres from digital data. Part I: development and operation of the scheme. Aust Meteor Mag. 39, 155-166

Nakamura H, Sampe T, Tanimoto Y, Shimpo AA (2004) Observed associations among storm tracks, jet streams and midlatitude oceanic fronts. In: Wang C, Xie SP, Carton JA (eds) Earth's climate: the ocean-atmosphere interaction, AGU Geophy. Monograph Series, 147:329-345

Neu U et al (2013) A community effort to intercompare extratropical cyclone detection and tracking algorithms. Bull Am Meteorol Soc 94(4):529-547. doi:10.1175/BAMS-D-11-00154.1

Pellerin P, Ritchie H, Saucier FJ, Roy F, Desjardins S., Valin M, Lee V (2004) Impact of a two-way coupling between an atmospheric and ocean-ice model over the Gulf of St. Lawrence. Mon Weather Rev 132:1379-1398

Pfahl S, Wernli H (2012) Quantifying the relevance of cyclones for precipitation extremes. J. Clim 25:6770-6780

Pinto JG, Spangehl T, Ulbrich U, Speth P (2006) Assessment of winter cyclone activity in a transient ECHAM4-OPYC3 GHG experiment. Meteorol Z 15:279-291

Pinto JG, Bellenbaum N, Karremann MK, Della-Marta PM (2013) Serial clustering of extratropical cyclones over the North Atlantic and Europe under recent and future climate conditions. J Geophys Res Atmos 118, 12476-12485, doi:10.1002/2013JD020564

Radojevic M (2006) Activité des cyclones extra-tropicaux simulés par le modèle canadien de circulation général. Mémoire de maitrise, Sciences de l'Atmosphère. Université du Québec à Montréal, Montréal, pp 119

Reitan CH (1974) Frequencies of cyclones and cyclogenesis for North America, 1951-1970. Mon Weather Rev 102:861-868

Reynolds RW, Rayner NA, Smith TM, Stokes DC, Wang W (2002) An improved in situ and satellite SST analysis for climate. J Clim
15:1609-1625. doi:10.1175/1520-0442(2002)015\%3C1609:AIISA $\mathrm{S} \% 3 \mathrm{E} 2.0 . \mathrm{CO} ; 2$

Rosu C (2005) Les caractéristiques des cyclones et l'apport d'eau dans les bassins versants du Québec. Mémoire de maîtrise, Sciences de l'Atmosphère. Université du Québec à Montréal, Montréal, pp 118

Sanders F, Gyakum JR (1980) Synoptic-dynamic climatology of the "bomb". Mon Weather Rev 108(10):1589-1606

Seiler C, Zwiers FW (2016) How well do CMIP5 climate models reproduce explosive cyclones in the extratropics of the Northern Hemisphere? Clim Dyn 3(3-4):1241-1256

Šeparović L, Alexandru A, Laprise R, Martynov A, Sushama L, Winger K, Tete K, Valin M (2013) Present climate and climate change over North America as simulated by the fifth-generation Canadian regional climate model. Clim Dyn. doi:10.1007/ s00382-013-1737-5

Serreze MC (1995) Climatological aspects of cyclone development and decay in the Arctic. Atmos Ocean 33:1-23

Sinclair MR (1994) An objective cyclone climatology for the Southern Hemisphere. Mon Weather Rev 122:2239-2256

Sinclair RM (1997) Objective identification of cyclones and their circulation intensity, and climatology. Weather Forecast 12:595-612

Sisson PA, Gyakum JR (2004) Synoptic-scale precursors to significant cold-season precipitation events in Burlington. Vermont Wea Forecast 19:841-854

Stewart RE, Bachand D, Dunkley RR, Giles AC, Lawson B, Legal L, Miller ST, Murphy BP, Parker MN, Paruk BJ, Yau MK (1995) Winter storms over Canada. Atmos Ocean 33(2):223-247

Stull RB (2000) Meteorology for scientists and engineers: a technical companion book with Ahrens' Meteorology Today. Brooks/Cole, Boston

Trigo IF (2006) Climatology and interannual variability of storm-tracks in the Euro-Atlantic sector: a comparison between ERA-40 and NCEP/NCAR reanalyses. Clim Dyn 26:127-143. doi:10.1007/ s00382-005-0065-9

Ulbrich U, Fink AH, Klawa M, Pinto JG (2001) Three extreme storms over Europe in December 1999. Weather 56:70-80

Ulbrich U, Pinto J, Kupfer H, Leckebusch G, Spangehl T, Reyers M (2008) Changing Northern Hemisphere storm tracks in an ensemble of IPCC climate change simulations. J Clim 21(8):1669-1679

Verseghy LD (2008) The Canadian land surface scheme: technical documentation-version 3.4. Climate Research Division. Science and Technology Branch. Environment Canada

Vitolo R, Stephenson DB, Cook IM, Mitchell-Wallace K (2009) Serial clustering of intense European storms. Meteorol Z 18:411-424

von Salzen K, Scinocca JF, McFarlane NA, Li J, Cole JNS, Plummer D, Solheim L (2013) The Canadian Fourth Generation Atmospheric Global Climate Model (CanAM4). Part I: representation of physical processes. Atmos Ocean 51:104-125, doi:10.1080/07055900.2 012.755610

Wernli H, Dirren S, Liniger MA, Zillig M (2002) Dynamical aspects of the life-cycle of the winter storm "LOTHAR" (24-26 December 1999). Q J R Meteorol Soc 128:405-429

Wilcoxon F (1945) Individual comparisons by ranking methods. Biometrics $1: 80-83$

Woollings T, Hoskins B, Blackburn M, Hasssell D, Hodges K (2010) Storm track sensitivity to sea surface temperatures resolution in a regional atmosphere model. Clim Dyn 35:343-353

Zadra A, McTaggart-Cowan R, Roch M (2012) Recent changes to the orographic blocking. Seminar presentation, RPN, Dorval, Canada, 30 March 2012. http://collaboration.cmc.ec.gc.ca/science/rpn/ SEM/dossiers/2012/seminaires/2012-03-30/Seminar_2012-03-30_ Ayrton-Zadra.pdf. Accessed 19 Apr 2017

Zappa G, Shaffrey LC, Hodges KI (2013) The ability of CMIP5 models to simulate north Atlantic extratropical cyclones. J Clim 26(15):5379-5396 